\title{
Cyclotrons as Drivers for Precision Neutrino Measurements
}

\author{
A. Adelmann, ${ }^{1}$ J. Alonso, ${ }^{2}$ W. A. Barletta, ${ }^{2}$ J. M. Conrad, ${ }^{2}$ M. H. Shaevitz, ${ }^{3}$ J. Spitz, ${ }^{2}$ \\ M. Toups, ${ }^{2}$ and L. A. Winslow ${ }^{4}$ \\ ${ }^{1}$ Paul Scherrer Institute, 5232 Villigen, Switzerland \\ ${ }^{2}$ Massachusetts Institute of Technology, Cambridge, MA 02139, USA \\ ${ }^{3}$ Columbia University, New York, NY 10027, USA \\ ${ }^{4}$ University of California, Los Angeles, CA 90095, USA
}

Correspondence should be addressed to J. M. Conrad; conrad@mit.edu

Received 23 July 2013; Accepted 23 October 2013; Published 23 January 2014

Academic Editor: Leslie Camilleri

Copyright (C) 2014 A. Adelmann et al. This is an open access article distributed under the Creative Commons Attribution License, which permits unrestricted use, distribution, and reproduction in any medium, provided the original work is properly cited. The publication of this article was funded by $\mathrm{SCOAP}^{3}$.

As we enter the age of precision measurement in neutrino physics, improved flux sources are required. These must have a well defined flavor content with energies in ranges where backgrounds are low and cross-section knowledge is high. Very few sources of neutrinos can meet these requirements. However, pion/muon and isotope decay-at-rest sources qualify. The ideal drivers for decay-at-rest sources are cyclotron accelerators, which are compact and relatively inexpensive. This paper describes a scheme to produce decay-at-rest sources driven by such cyclotrons, developed within the DAE $\delta$ ALUS program. Examples of the value of the high precision beams for pursuing Beyond Standard Model interactions are reviewed. New results on a combined DAE $\delta$ ALUSHyper-K search for CP violation that achieve errors on the mixing matrix parameter of $4^{\circ}$ to $12^{\circ}$ are presented.

\section{Introduction}

As we reach the 100th anniversary of the birth of Bruno Pontecorvo, neutrino physics is facing a transition. Neutrino oscillations are well established, albeit in a different form from what Pontecorvo expected $[1,2]$. We have a data-driven "Neutrino Standard Model," ( $(2 \mathrm{SM})$ which, despite questions about its underlying theoretical description, is remarkably predictive. Now, the neutrino community must pivot from "searches" to "precision measurements," in which we can test the $\nu \mathrm{SM}$. The transition requires new and better tools for these measurements and further calls for original approaches to experiments.

The $\nu \mathrm{SM}$ is simply described in Figure 1. The three known neutrino flavors mix within three mass states. The separations between the states, or "mass splittings," are defined as $\Delta m_{i j}^{2}=m_{i}^{2}-m_{j}^{2}$, for $i, j=1, \ldots, 3$. The historical name for the smaller splitting $\left(\Delta m_{21}^{2}\right)$ is $\Delta m_{\text {sol }}^{2}$ and the larger mass splitting $\left(\Delta m_{31}^{2} \approx \Delta m_{32}^{2}\right)$ is referred to as $\Delta m_{\mathrm{atm}}^{2}$, in honor of the solar and atmospheric experiments that established the existence of each. The early solar [3-6] and atmospheric [7-9] experiments have been joined by new results [10-15] to establish this phenomenology [16].

The mixings are described with a $3 \times 3$ matrix, commonly called the Pontecorvo-Maki-Nakagawa-Sakata (PMNS) matrix, that connects the mass eigenstates $\left(v_{1}, v_{2}\right.$, and $\left.v_{3}\right)$ to the flavor eigenstates $\left(v_{e}, v_{\mu}\right.$, and $\left.v_{\tau}\right)$ :

$$
\begin{aligned}
U & =\left(\begin{array}{lll}
U_{e 1} & U_{e 2} & U_{e 3} \\
U_{\mu 1} & U_{\mu 2} & U_{\mu 3} \\
U_{\tau 1} & U_{\tau 2} & U_{\tau 3}
\end{array}\right) \\
& =\left(\begin{array}{lll}
0.795-0.846 & 0.513-0.585 & 0.126-0.178 \\
0.205-0.543 & 0.416-0.730 & 0.579-0.808 \\
0.215-0.548 & 0.409-0.725 & 0.567-0.800
\end{array}\right),
\end{aligned}
$$

where the ranges indicate our knowledge of each of the entries [27]. Together with the mass splittings, the mixing matrix is pictorially represented in Figure 1, in which the lengths of the colored bars are proportional to the squared moduli of the matrix elements, $\left|U_{\alpha i}\right|^{2}$. 


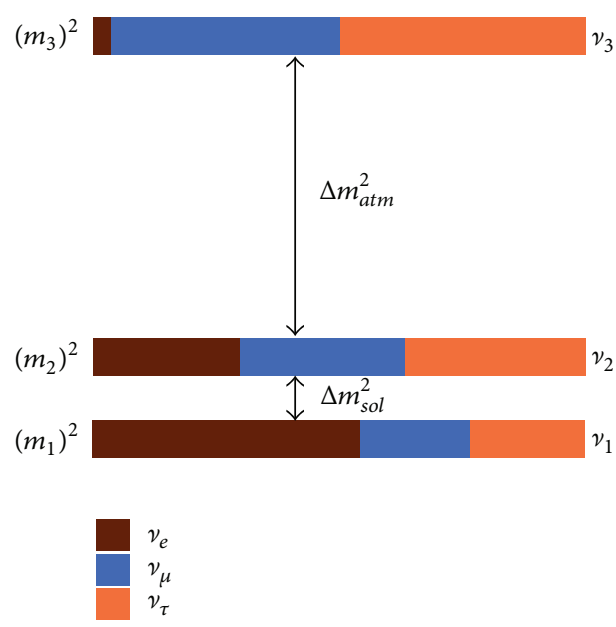

FIGURE 1: Illustration of the " $\nu \mathrm{SM}$ " showing mass states and mixings. Note that this drawing depicts only one possible mass ordering. There remain many open questions that surround this data-driven picture of neutrinos and oscillations.

As can be seen, our current knowledge of the mixing matrix values is imprecise. The last entry, $U_{e 3}$, was found to be nonzero just two years ago [28-31]. Our current state of measurement of the neutrino mixing matrix is analogous to that of the quark sector in 1995 [32], immediately after the discovery of the top quark. Unlike in the quark sector and its utilization of strong production, neutrino physicists are faced with the difficulty of both weak production and weak decay. Our route to precision therefore drives us to high-intensity sources coupled with ultralarge detectors.

Even at this relatively early stage, the $\nu \mathrm{SM}$ has been remarkably predictive. For example, the $\left|U_{e 3}\right|$ element was found with the $\Delta m_{\mathrm{atm}}^{2}$ splitting [33] as expected from the model. However, many open questions remain. For example, we know the sign of the squared mass splitting between the two states involved in solar oscillations [16], but not the sign involved in atmospheric oscillations. Figure 1 shows a "hierarchy" of the mass states, after arranging the large and small splittings so that the orientation is consistent with what is seen in the quark sector. It is unclear if the neutrinos are oriented in this "normal hierarchy" $\left(m_{1}<m_{2}<m_{3}\right)$, as shown, or if the orientation is actually "inverted" $\left(m_{3}<\right.$ $\left.m_{1}<m_{2}\right)$. Further, although the magnitudes of each of the splittings have been measured, the absolute mass of the neutrino is not known. We know that there is a $3 \times 3$ matrix that describes the mixing but we do not know if there is $\mathrm{CP}$ violation present as in the quark sector. There are also more exotic questions surrounding the neutrino and oscillations. For example, are there new forces appearing in neutrino interactions and oscillations? Do exotic noninteracting ("sterile") neutrinos mix with the known active flavors? Hints for all of these possibilities exist [34-38] with evidence extending up to $4 \sigma$. The next generation of neutrino experiments must investigate these results and clarify the present picture. If history is any indicator of the future, it is quite likely that these experiments, along with the more conventional ones within the $\nu \mathrm{SM}$, will raise even more surprises.
The next step in neutrino physics requires improved tools, in particular, sources from which the energy distribution and flavor content are very well defined. The beam energy must be in a range where the neutrino interaction cross-section is understood, backgrounds are low, and the detectors are highly efficient. Decay-at-rest (DAR) sources satisfy these requirements and provide an opportunity for the precision measurements required for the future of neutrino physics.

This paper explores cyclotrons as a relatively low cost means of producing DAR sources at or near underground laboratories. We begin by discussing the pros and cons of DAR for neutrino physics. Next, we review the history of and development of cyclotrons. We then describe the twocyclotron system under development within the DAE $\delta$ ALUS program. This is a phased program which ultimately results in three sites that produce DAR fluxes, located near an ultralarge detector. The final sections of this paper provide examples of the precision science opened up by the DAR sources. We explore tests in neutrino oscillations and neutrino scattering. We also discuss the potential impact beyond particle physics.

\section{Decay-at-Rest Sources of Neutrinos}

The most common source presently used in neutrino physics is the "conventional muon-neutrino beam." Such a source is produced with $\mathrm{GeV}$-scale protons striking a target, resulting in pions and kaons which decay-in-flight (DIF) to produce neutrinos. The energy distribution and relevant backgrounds of a given DIF beam are dependent on the design of the beamline. For example, the characteristics of a beam are quite sensitive to the magnetic focusing and decay region geometry as well as primary/secondary hadron production and interaction physics in the target. These complications make first principles predictions of the flavor-dependent neutrino energy distributions for both signal and background from DIF beams quite difficult.

A number of techniques are available for DIF-based experiments to understand the neutrino flux. Experiments with very high interaction rates can use data to constrain the flux. For example, the MiniBooNE experiment has successfully used $v_{\mu}$ interactions, which come largely via pion DIF from the Booster Neutrino Beamline (BNB) at Fermi National Laboratory, to constrain the $v_{e}$ backgrounds that are due to the subsequent decay of the pion's daughter muon [39]. Long baseline experiments use both a near detector and a far detector to reduce flux uncertainties. This comparison works well for charged current interactions, in which the neutrino energy can be fully reconstructed. Neutrinos which are within the acceptance of the far detector are considered in measuring the flux of the near detector. However, this approach is significantly less precise for neutral current background events, because the neutrino energy cannot be fully reconstructed. Lastly, a wide range of event topologies are produced by conventional beams, which range in energy from hundreds of $\mathrm{MeV}$ (e.g. JPARC [40], BNB [39]) up to tens of $\mathrm{GeV}$ (e.g. NuMI [41], CNGS [42]). The crosssections for neutral current events and topologies involving pion production are not well measured and understood [43] 


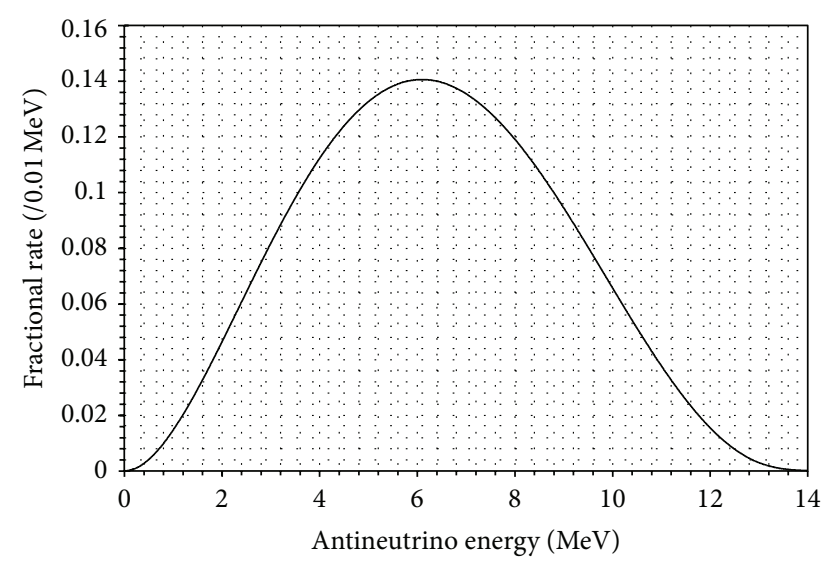

Figure 2: The ${ }^{8} \mathrm{Li}$ isotope DAR anti-electron-neutrino flux.

and the pions (and their decay products) produced in the events can lead to electron-like backgrounds.

DAR neutrino sources, produced through $\pi \rightarrow \mu \rightarrow$ $v$ and isotope decay, offer a precision alternative to DIF beams. DAR flux sources have well defined flavor content and energy distributions, as can be seen with the flux from a $\pi \rightarrow \mu \rightarrow \nu$ source shown in Figure 3. However, the neutrino energy is low compared to DIF-ranging from a few $\mathrm{MeV}$ to $52.8 \mathrm{MeV}$. The low energy of a DAR source is both an advantage and a disadvantage. A great advantage is that two of the best-known neutrino cross-sections, each with less than $1 \%$ uncertainty, are accessible at DAR beam energies. The first is the inverse beta decay interaction (IBD, $\left.\bar{v}_{e}+p \rightarrow e^{+}+n\right)$. This has a cross-section that is well known because it is connected to neutron decay, which is measured very precisely [46]. IBD can be efficiently observed by requiring a coincidence between the prompt positron and delayed neutron capture signal [12, 28-30]. The coincidence signature also allows the signal to be easily distinguished from background, especially, if the background is low as is the case for an underground detector. The second is neutrino electron elastic scattering $(v+e \rightarrow v+e)$. This cross-section is well constrained by Standard Model measurements of $e^{+} e^{-}$ scattering [47]. Although this interaction lacks a coincidence signal, it is highly directional, even at DAR energies. On the other hand, the low energy neutrinos from a DAR source means that the relevant interactions have low absolute crosssections, leading to the high flux requirement. A DAR source therefore has the overall disadvantage of requiring a very intense source that can be installed at or near a detector in an underground location. Also, DAR fluxes are isotropic, and, depending on the $L / E$ demanded by the experiment, the detector may subtend small solid angle. Below, we will show that cyclotrons, as DAR neutrino drivers, have sufficiently high intensity and small enough size to overcome these disadvantages.

DAR sources range in energy from up to a few $\mathrm{MeV}$ from isotope decay, where we use ${ }^{8} \mathrm{Li}$ decay as our example (see Figure 2), to $52.8 \mathrm{MeV}$ from the $\pi^{+} \rightarrow \mu^{+}$chain (see Figure 3). The flux from isotope decay is pure in flavor, while the pion/muon decay has well defined flavor ratios.

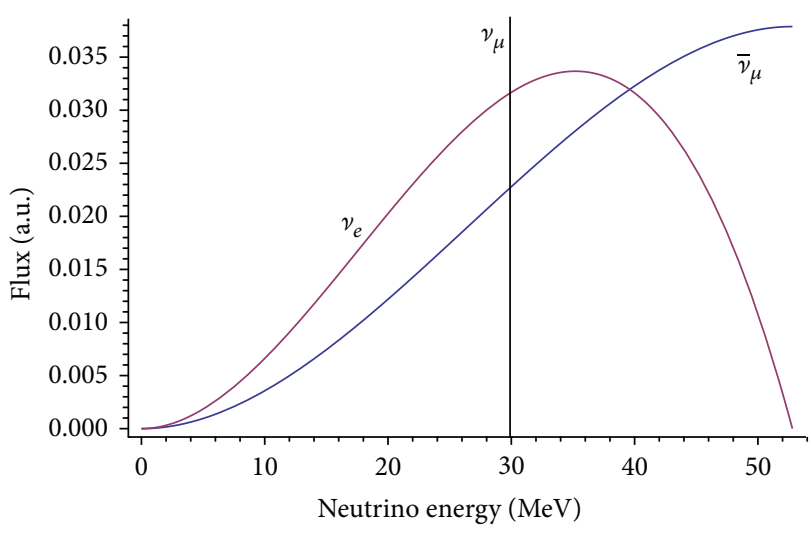

FIGURE 3: Neutrino flux distribution from a pion/muon DAR source, from [17].

The pion/muon DAR beam is best produced by impinging low energy $\sim 800 \mathrm{MeV}$ protons on a light target to produce a high rate of pions through the $\Delta$-resonance. The target must be surrounded by heavy material to stop the outgoing pions before DIF. In this case, the neutrinos originate primarily from $\pi^{+}$or $\mu^{+}$decay. The negatively charged pions and muons stop and capture on nuclei before they can decay to produce neutrinos [48]. The production of kaons or heavier mesons, which could produce unwanted backgrounds, is negligible if the primary proton energy is below about $1 \mathrm{GeV}$. The $\bar{v}_{e}$ flux can be maintained at the level $\sim 5 \times 10^{-4}$ of the $\bar{v}_{\mu}$ flux in the $20<E_{v}<52.8 \mathrm{MeV}$ energy range. As a result, the source is well suited to search for $\bar{\nu}_{e}$ appearance through oscillations [49], as discussed below.

An isotope DAR source produces a pure electron flavor flux through $\beta$-decay. Such a source can be produced through high-intensity, low-energy $(60 \mathrm{MeV} / \mathrm{n})$ protons impinging on a beryllium target. These and subsequent interactions result in a flood of neutrons that are captured on surrounding material to produce the isotope of interest. Precision experiments are best performed using neutrinos above $3 \mathrm{MeV}$, where environmental backgrounds are low. As a result, high $Q$ value isotopes are favored. This process produces a very pure $\bar{v}_{e}$ flux of well defined energy that can be used for scattering and neutrino disappearance experiments.

Below, we discuss the use of ${ }^{8} \mathrm{Li}$ as the decaying isotope, produced by neutron capture in a $99.99 \%$ pure ${ }^{7} \mathrm{Li}$ sleeve surrounding the target. The ${ }^{6} \mathrm{Li}$ content must be removed because this isotope has a high neutron capture cross-section that competes with the ${ }^{7} \mathrm{Li}$. Lithium was chosen because $99.99 \%$ isotopically pure ${ }^{7} \mathrm{Li}$ is used by molten salt reactors and hence can be obtained. We examined other isotopes, including, in particular, ${ }^{11} \mathrm{~B}$ which can produce ${ }^{12} \mathrm{~B}$ through neutron capture. However, in this case, we could not find commercially available ${ }^{11} \mathrm{~B}$ at sufficient purity to achieve a high flux.

The IBD interaction has a large cross-section at these $\mathrm{MeV}$-scale energies, as shown in Figure 4. We note, however, that, as the IBD interaction requires free protons as an interaction target, this approach is relevant for water- and scintillator-based detectors only. The IBD interaction has 


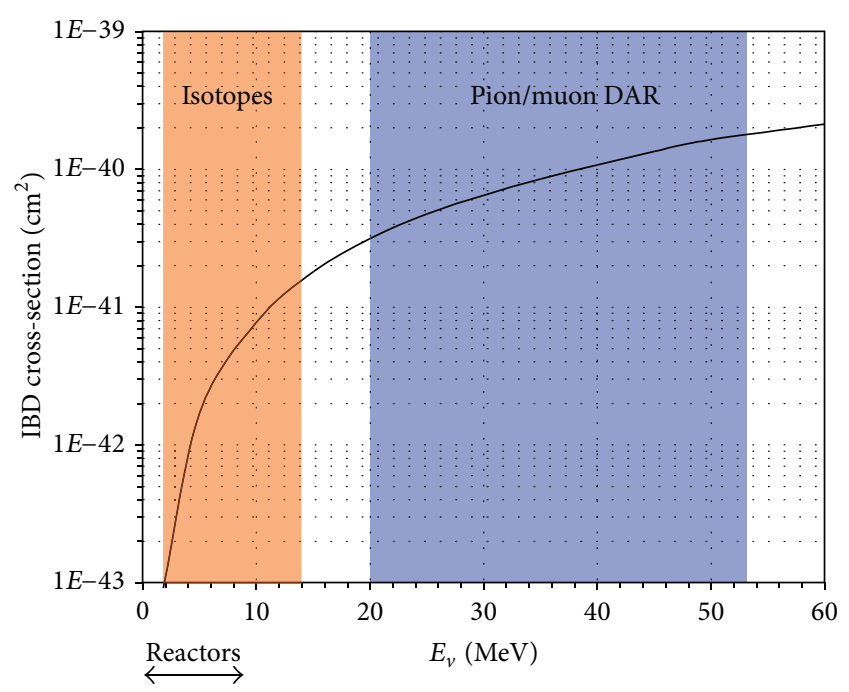

FIGURE 4: Overlay of high flux regions on the IBD cross-section.

a distinguished history. Pontecorvo himself first suggested a search for this interaction, in a 1946 report to the National Research Council of Canada [50,51], and it was the first type of neutrino interaction observed [52]. IBD is the signal interaction now widely used in reactor experiments. The reactor IBD range is shown in Figure 4 in comparison to the DAR fluxes.

\section{Cyclotrons as DAR Source Drivers}

Cyclotrons represent ideal drivers for the DAR sources discussed above. These machines are compact and of low cost compared to most particle physics accelerators. The size, power, and cooling requirements are sufficiently modest that it is possible to install cyclotrons at underground laboratories where these sources can be paired with existing large scintillator and water detectors. Cyclotrons date back to Pontecorvo's era. However, and as in the case of neutrino physics, cyclotrons have come a long way since their origin. Modern cyclotrons are capable of producing the very high intensity flux required for modern precision neutrino measurements.

This section reviews the history of cyclotrons, with some discussion about how cyclotrons work. We consider important examples in use today and then discuss their development within the DAE $\delta$ ALUS project.

3.1. A Brief History of Cyclotrons. Unbeknownst to Ernest O. Lawrence, the cyclotron was first invented by Leo Szilard, who received a German patent for the device in 1929, but Szilard never attempted a practical realization of his idea. Lawrence's own invention stemmed from his study of a paper by Rolf Wideroe on resonant acceleration in linear structures using radio frequency (RF) voltages. Although Lawrence could not understand German, he was able to understand enough of the concept from the drawings and equations to come to his own invention.

In the Wideroe linear accelerator, a beam of ions was accelerated through a series of small gaps between hollow metal tubes, called drift tubes, connected in series to the radio frequency $(\mathrm{RF})$ voltage generator. At any instant of time successive gaps carry a voltage of opposite sign-the voltage between successive gaps is shifted by 180 degrees of RF phase. While traversing the drift tubes the ions are shielded from the time-varying electric field. The length of each drift tube is chosen so that the voltage changes sign during the time it takes the ion to traverse the tube, allowing the ion to increase its energy. Since the nonrelativistic ions increase their velocity in passing through the gaps, each successive drift tube must be longer (proportional to the ion velocity times the RFwavelength) for the ion motion to remain in synchronism with the RF generator. Higher and higher energy meant that the length of the accelerator increased nonlinearly, at least initially. The final beam energy is just the RF voltage times the number of gaps.

To reach energies of $1 \mathrm{MeV}$ or more, Lawrence's insight was that, for nonrelativistic particles injected into a dipole magnetic field perpendicular to the particle velocity, the revolution frequency is independent of the particle energy. Higher energy particles travel on larger orbits, maintaining synchronous (or isochronous) motion. If the ion orbits are contained within two hollow "D"-shaped electrodes ("dees") which are connected to an RF-voltage source, one can accelerate ions to energies not possible with DC-voltage structures. Beam is injected at the center of the device and spirals outward.

Lawrence's ideas were soon realized in practice for protons by his student M. Stanley Livingston. As has been the case since the first accelerators, the Lawrence team pushed forward on two fronts: particle energy and beam intensity. The strong limitations on intensity were imposed by losses of ions on the vertical surfaces of the cyclotron's vacuum chamber. This problem was mitigated when magnetic field shims were introduced to provide a radial component to the magnetic field. The radial component increased with distance from the center of the cyclotron. The result was a vertical restoring force (vertical focusing) that confines the beam to the median horizontal plane. With vertical focusing, many successive bunches of beam particles, each with a different kinetic energy, could be accelerated in the cyclotron; that is, the Lawrence cyclotron delivered a continuous train of bunches of ions spaced by one RF period. In fact, the synchronism between ion motion and the RF voltage only requires that the revolution period equals an integer times the RF-period. Any odd integer, $N$, will work. Choosing $N>1$ allows even more bunches in the cyclotron at any one time; even high beam currents are possible.

Trying to increase the proton energy significantly beyond $10 \mathrm{MeV}$ provided a different difficulty. The revolution frequency (cyclotron frequency) began to decrease due to the relativistic increase in the proton mass. Even a change as small as 1 to $2 \%$ is enough for the revolution frequency to be outside of the frequency bandwidth of the RF generator and therefore to lose synchronism with the RF voltage. The solution to this limitation seems simple: modulate the RF to lower values to maintain synchronism with the highestenergy beam particles. The frequency modulated cyclotron (or synchrocyclotron) allowed the Lawrence team to achieve 
energies as high as $340 \mathrm{MeV}$ with their 184-inch cyclotron. At the University of Chicago, Enrico Fermi's team reached $450 \mathrm{MeV}$ with the slightly smaller 170 inch cyclotron. In the early 1950s this machine produced copious pions via the $\Delta(3,3)$-resonance. The age of accelerator-generated neutrinos had begun. It is noteworthy that Fermi and Szilard were also responsible for the invention of another important source of man-made neutrinos: the nuclear reactor.

Even larger machines were built in Russia. Russia's effort culminated in the giant $1 \mathrm{GeV}$ proton synchrocyclotron at the Leningrad Institute for Nuclear Physics in Gatchina. This machine was built around the world's largest one-piece electromagnet with a pole diameter of $7 \mathrm{~m}$ and iron weight of almost 8000 tonnes. Maintaining synchronism through frequency modulation had come at a price. Only one bunch, that is, protons of only a single energy, could be accelerated at any one time. Therefore, the Gatchina machine could only accelerate $0.2 \mu \mathrm{A}$, hundreds of times less than classical fixedfrequency cyclotrons.

Generating continuous trains of ion bunches at energies exceeding $10 \mathrm{MeV}$ per nucleon required yet another invention that would preserve particle synchronism despite the acceleration being provided by fixed frequency RF power. The solution published by Thomas in 1938 [53] showed that conditions for both synchronism and vertical focusing could be maintained if the vertical magnetic field, $B_{\theta}$, varied with polar angle. This variation (so-called hills and valleys of the magnetic field or field flutter) introduces a radial component to the velocity, $v_{r}$, of the ions and therefore an additional focusing effect due to the cross product $\mathbf{B}_{\theta} \times \mathbf{v}_{\mathbf{r}}$. This combination leads to scalloped orbits rather than simple spirals. If, in addition to the azimuthal variation, the vertical field increases in strength at larger radii, the revolution period can remain constant (isochronous) independent of energy of the ions over the full range of operation of the cyclotron.

Donald Kerst introduced a further improvement of Thomas' scheme of azimuthal variation by radial sectors. Kerst suggested using spiral sectors to increase axial focusing of the beam even more through the application of the alternating gradient principle, which was by then being designed into synchrotrons. Spiral sectors are now used in almost all cyclotrons over $\sim 40 \mathrm{MeV}$, enormously increasing both the energies and the intensities available and thereby providing a factor of $\sim 1000$ more intense beams for $\pi, \mu, \mathrm{n}$, and neutrino production at low energies.

The energy and intensity (or current) range provided by historical and present-day cyclotrons, compared to other types of circular accelerators, is shown in Figure 5. Various types of cyclotrons are noted: FF is the fixed field or classical cyclotron; FM is the frequency modulation (synchro-) cyclotron; and AVF is the azimuthal varying field cyclotron. One can see that, at the low energies needed for DAR beams, cyclotrons are ideal drivers. Linear accelerators are also an option but require much higher power and have much higher cost per unit energy than cyclotrons.

Research on two existing cyclotrons has provided important intellectual input for the $800 \mathrm{MeV}$ machine ultimately envisioned for the DAE $\delta$ ALUS cyclotron program. These are the separated-sector ring cyclotron at the Paul Scherrer

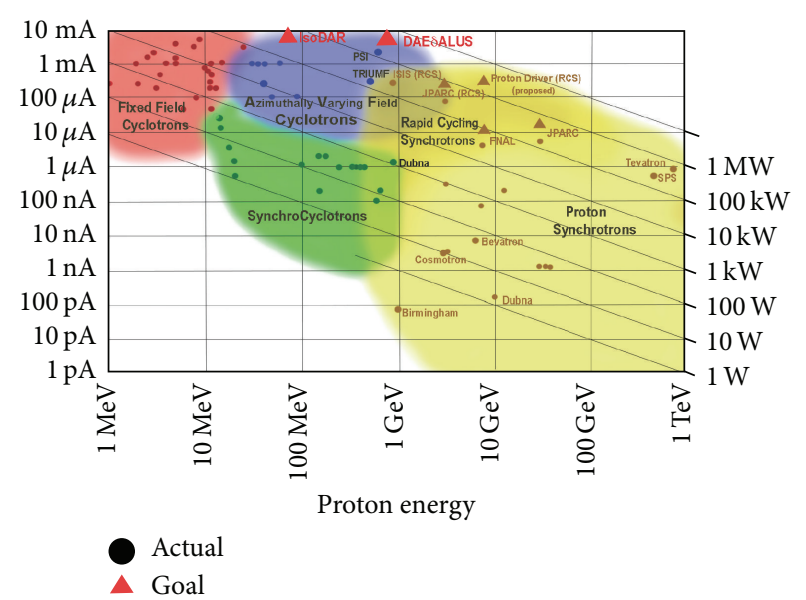

FIGURE 5: Proton energy versus current for various existing machines. The type of accelerator is indicated. Various types of cyclotrons are noted, where FF is the fixed field or classical cyclotron; FM is the frequency modulation (synchro-) cyclotron; and AVF is the azimuthal varying field cyclotron. This plot is taken from [18].

Institute (PSI) in Villigen, Switzerland [54], and the superconducting ring cyclotron (SRC) at RIKEN, Wako, Japan [55]. In separated-sector cyclotrons, the sectors have individual yokes and coils; the valleys are magnetic field-free and are available for RF power, beam injection and extraction, and diagnostics. Such machines require a medium energy (several tens of $\mathrm{MeV}$ ) injector. With an energy of $590 \mathrm{MeV}$ and beam current of $2.4 \mathrm{~mA}$, the PSI ring cyclotron is currently the world's most powerful accelerator in this energy range, delivering 1.4 MW of protons [54], as seen in Figure 5. The PSI complex routinely achieves $99.98 \%$ extraction efficiency, and this sets the bar for future accelerators such as those in the DAE $\delta$ ALUS program.

The RIKEN SRC is the world's first ring cyclotron that uses superconducting magnets, and it has the strongest beam bending force among the cyclotrons. This cyclotron, although designed for high-energy highly stripped heavy ion beams, represents an engineering "proof-of-practice" design for a cyclotron magnet applicable for the $800 \mathrm{MeV} / \mathrm{n}$ DAE $\delta$ ALUS SRC. RIKEN does not appear on Figure 5 because it is a heavy ion rather than a proton machine. As such, the current from the RIKEN machine is limited by the available shielding, and not by the machine design. RIKEN can boost the ion beam energy up to $440 \mathrm{MeV} /$ nucleon for light ions and up to $350 \mathrm{MeV} /$ nucleon for very heavy ions, such as uranium nuclei, to produce intense radioactive beams. The SRC consists of six major superconducting sector magnets with a maximum field of $3.8 \mathrm{~T}$. The total stored energy is $235 \mathrm{MJ}$, and its overall dimensions are $19 \mathrm{~m}$ diameter, $8 \mathrm{~m}$ height, and 8,300 tons. The magnet system assembly was completed in August 2005 and successfully reached the maximum field in November 2005. After magnetic field measurements for two months, the superconducting magnet was installed and the first beam was extracted from the SRC in December 2006.

3.2. Cyclotrons as Pion/Muon Factories. Cyclotrons have been used to produce pions and muons for many years; what 


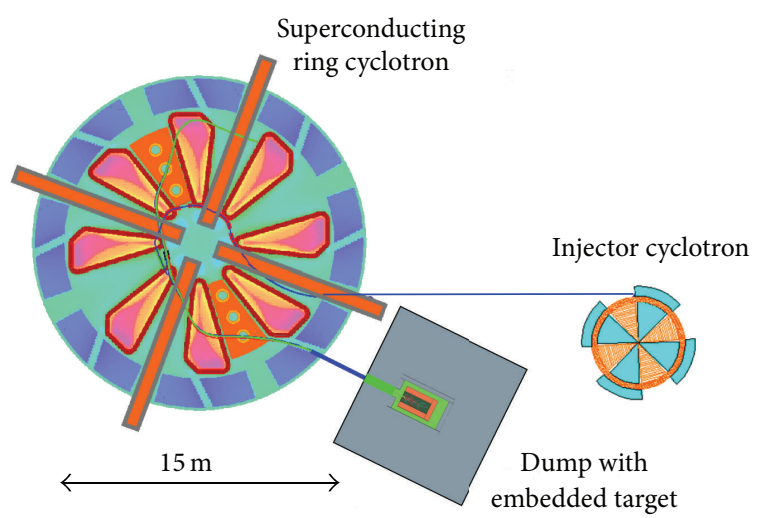

Figure 6: A DAE $\delta$ ALUS module.

is novel about DAE $\delta$ ALUS is their application as drivers for DAR sources, in which the pions and muons come to rest and decay to neutrinos. In fact, two out of three of the major "meson factories" commissioned in the 1970s were cyclotron-based. These were TRIUMF, in Vancouver, BC, Canada [56], and SIN [57] (now PSI) in Villigen, Switzerland. The competing technology was LAMPF [58] (now LANSCE) at Los Alamos, which was an $800 \mathrm{MeV}$ linear accelerator. These two cyclotron facilities remain at the forefront of precision pion and muon studies to this day. TRIUMF, a $500 \mathrm{MeV} \mathrm{H} \mathrm{H}^{-}$cyclotron, produces several hundred $\mu \mathrm{A}$. This program has expanded to also become a world-leading laboratory for radioactive ion beams. PSI is currently the world's most powerful accelerator in this energy range with $590 \mathrm{MeV}$ protons. At $1.4 \mathrm{MW}$ of beam power, this cyclotron is a shining example of high-quality beams with extremely high extraction efficiency (99.98\%) and low beam losses ( $<200 \mathrm{~W}$ per cyclotron vault). An example of the beautiful muon physics now being published from PSI is the precision measurement of $G_{F}$ from the MuLan experiment [59]. However, the PSI program with the primary beam is now evolving toward being primarily directed at production of low-energy ( $\mathrm{meV}$ ) neutrons via the spallation process for neutron scattering and diffraction studies of materials. These cyclotrons, which have been a tremendous asset to the field, inform the DAE $\delta$ ALUS design below.

Although neither the TRIUMF nor PSI machines have been applied to the neutrino field, DAR experiments were conducted at Los Alamos with the competing LAMPF beam $[60,61]$. Also, it should be noted that other low-energy synchrotrons have also hosted or are considering important neutrino decay-at-rest experiments, namely, the $700 \mathrm{MeV}$ ISIS machine at the Rutherford Appleton Lab in UK (KARMEN [62]) and the SNS at Oak Ridge [63].

\subsection{The DAE $\delta A L U S$ Cyclotrons. The DAE $\delta A L U S$ cyclotron} system accelerates ions through a series of two cyclotrons. The full system is called an "accelerating module." Figure 6 shows the schematic layout of one of the $\mathrm{H}_{2}^{+}$accelerating modules. The DAE $\delta$ AUS injector cyclotron (DIC) captures up to $5 \mathrm{~mA}$ (electrical) of $\mathrm{H}_{2}^{+}$and accelerates the beam to about $60 \mathrm{MeV} / \mathrm{n}$. This beam is extracted electrostatically. This beam can then be used for a stand-alone program (IsoDAR) or for injection into the DSRC for further acceleration (DAE $\delta$ ALUS). This second machine consists of 8 wedgeshaped superconducting magnets and $6 \mathrm{RF}$ cavities (4 of the PSI single-gap type, 2 double-gap). The stripper foil is located at the outer radius, at the trailing edge of one of the sector magnets, and the extraction channel comes out roughly along one of the valleys about $270^{\circ}$ away. Figure 6 also shows schematically one of the beam dumps, a graphite block with a hole shaped to correspond to the beam profile so the energy is uniformly distributed over a wide area. The graphite is surrounded by a copper, water-cooled jacket and is expected to dissipate $6 \mathrm{MW}$ of beam power.

A key aspect of the design is acceleration of $\mathrm{H}_{2}^{+}$. This novel choice of ion was selected by Calabretta [64] in response to a suggestion by Rubbia in the 1990s to use high-current, $\sim 1 \mathrm{GeV}$ cyclotrons for driving thorium reactors [65]. Since most cyclotrons accelerate protons or $\mathrm{H}^{-}$, the pros and cons of this choice are worth examining.

A drawback of $\mathrm{H}_{2}^{+}$is that the higher rigidity of this ion $(q / A=0.5)$ compared to bare protons or $\mathrm{H}^{-}$(both with $|q| / A$ $=1$ ) requires a cyclotron of relatively large radius. However, the size of the machine is practical given the higher fields available from superconducting magnets. In fact, the RIKEN $\mathrm{SRC}$ is close to the field and size specifications required.

By choosing the $\mathrm{H}_{2}^{+}$ion, one can use a stripping foil to cleanly extract the beam from the cyclotron. Although stripping extraction is not available to proton accelerators, it has been used extremely effectively in lower-energy cyclotrons that accelerate $\mathrm{H}^{-}$beams. The value of stripping extraction is that turn separation is no longer an issue. All ions will pass through the stripper foil, even if turn separation is not clean. Ions will be stripped from several turns, probably not more than two or three, and the protons will carry the energy associated with their turn number. The extraction channel, which will pass through the central region of the DSRC (as the protons are bent in rather than out as is the case with $\mathrm{H}^{-}$), will need adequate momentum acceptance to transmit all the protons from the turn where they are stripped.

It is the lower binding energy $(0.7 \mathrm{eV})$ of the $\mathrm{H}^{-}$ion that renders it unusable in a high-energy machine like the DSRC. This makes $\mathrm{H}^{-}$susceptible to Lorentz stripping in fields as low as $2 \mathrm{~T}$ and energies below $70 \mathrm{MeV}$. The higher binding energy of the $\mathrm{H}_{2}^{+}$ion $(2.7 \mathrm{eV}$, at least in its ground state) renders it more stable and able to survive to $800 \mathrm{MeV}$ in the highest $6 \mathrm{~T}$ fields anticipated in the DSRC.

Lastly, a great advantage of $\mathrm{H}_{2}^{+}$is reduction of spacecharge effects. The space charge of the particle beam produces a repulsive force inside the beam, which generates detuning effects. A measure of the strength of this effect, the "generalized perveance," is defined by

$$
K=\frac{q I}{2 \pi \epsilon_{0} m c^{3} \gamma^{3} \beta^{3}},
$$

where $q, I, m, c, \gamma$, and $\beta$ are the charge, current, rest mass, speed of light, and the relativistic parameters of the particle beam, respectively [66]. The higher the value of $K$ is, the stronger the space-charge detuning effects are. 
According to (2), the space-charge effects for the $5 \mathrm{~mA}$ of $\mathrm{H}_{2}^{+}$beam in the DSRC are equivalent to a $2.5 \mathrm{~mA}$ proton beam with the same $\gamma$. Consequently, they are similar to the space-charge effects present in the $2.4 \mathrm{~mA}$ proton beam currently being accelerated at PSI. Another degree of freedom to reduce space-charge effects is the choices injection energy and acceleration voltage. Given this premise, we have carried out precise beam dynamics studies, including the 3D spacecharge effects (excluding the central region of the DIC), with self-consistent $3 \mathrm{D}$ models implemented in the code Object Oriented Parallel Accelerator Library (OPAL) [67]. The beam dynamics model is described in detail in $[68,69]$. For the DSRC, we have implemented a simple stripper model into OPAL in order to study the complex extraction trajectories of the stripped protons.

DAE $\delta$ ALUS R\&D related to $\mathrm{H}_{2}^{+}$acceleration has begun at a test stand at Best Cyclotrons, Inc., in Vancouver, BC, Canada. These studies employ the VIS, or Versatile Ion Source, a nonresonant Electron Cyclotron Resonance (ECR) source [70] built at the Laboratori Nazionali del Sud (LNS) in Catania, Italy, which has been shipped to Vancouver for these tests. As with any ion source, protons, $\mathrm{H}_{2}^{+}$, and even $\mathrm{H}_{3}^{+}$will be emitted. We have begun studies of emittance, inflection into the cyclotron, capture, and acceleration of $\mathrm{H}_{2}^{+}$.

In summary, the DAE $\delta$ ALUS program has developed a plan for the production of two high power cyclotrons, one producing beam at $60 \mathrm{MeV} / \mathrm{n}$ and the other at $800 \mathrm{MeV} / \mathrm{n}$. The former provides the injector to the latter, which then can produce pion/muon DAR neutrino fluxes. As discussed below, the injector can also be used by itself to produce isotope DAR beams.

\subsection{Application of the DIC Cyclotron to Isotope Production.} The remainder of this paper will describe the value of the DAE $\delta$ ALUS cyclotrons for basic research in neutrino physics. However, it is worth pausing to note that these cyclotrons have practical applications. This has attracted industry involvement in DAE $\delta$ ALUS development. Examples range from medical applications to accelerator driven systems for thorium reactors. We concentrate on the former here and note that [71] provides a more extensive description of isotope production for medicine using the DIC cyclotron.

Even from Pontecorvo's days, isotopes produced by cyclotrons were being investigated for suitability in medical diagnostics and therapeutics. The first direct therapeutic use of beams occurred in 1937 with trials using neutrons produced from the $60 \mathrm{MeV}$ Crocker Cyclotron at Berkeley. Incidentally, this machine is still in use, primarily for proton treatments of eye tumors at UC Davis. Higher energy cyclotrons were built in the 1940's, the $800 \mathrm{MeV}$ 184-inch synchrocyclotron at Berkeley, and two similar-sized machines at Dubna and St. Petersburg. Medical treatments played prominent roles in all three machines, starting with stereotactic microsurgery with very fine beams for pituitary ablation. Then, when diagnostic tools such as CT scanners became available that could carefully measure the density of material upstream of a tumor to accurately predict the stopping point of the beam, Bragg-peak therapy with alpha-particle beams was instituted at the 184 inch Synchrocyclotron.

Cyclotrons are now used extensively for therapy with proton beams, with several commercial companies marketing highly effective cyclotron-based systems for proton therapy with beam energies of around $250 \mathrm{MeV}$ [72]. As beam currents needed for radiotherapy are only in the nA range, even with the losses inherent in degrading the fixed-energy beams down to match the required range in the patient, adequate beam brightness can be achieved with beams of no more than a $\mu \mathrm{A}$ of protons extracted.

Meanwhile, radioactive isotopes produced with cyclotrons of energies of $30 \mathrm{MeV}$ or less have become widely used in medical diagnostics and therapy, with an ever-increasing demand as techniques are refined and results improved. Beam currents in the range of $750 \mu \mathrm{A}$ to $2 \mathrm{~mA}$ are now being extracted from commercial and research isotope-producing cyclotrons; the limiting factor is often heat-dissipation in the complex targets that are needed for effective isotope production. Increased production capacity is being obtained by multiple extraction ports enabled by acceleration of $\mathrm{H}^{-}$ beams that can be extracted by stripping. Sharing the total beam power between two target stations enables greater production capacity.

The DAE $\delta$ ALUS injector cyclotron, used for IsoDAR, will become a powerful tool for isotope production along two different directions. As a source of $60 \mathrm{MeV}$ protons at $600 \mathrm{~kW}$, beam powers are substantially higher than existing isotope machines. This could enable either significantly greater yield on a single target, should the technology be developed to use all this beam power on a single target or increased versatility of the isotope factory by sharing the beam between many targets. As $\mathrm{H}_{2}^{+}$ions are extracted from the cyclotron via a conventional septum, a narrow stripper can be placed over a portion of the beam to convert ions passing through the stripper into protons that can then be cleanly separated from the body of the beam and transferred to a production target. The remaining beam is transported to further stripping stations, each peeling off a small portion of the beam to deliver it to a different target. In this way the power limits on any given target will not be exceeded, and high efficiency for use of the whole beam is maintained. Examples of the isotopes which can be produced, and their applications, are shown in Table 1.

A second isotope application of the $\mathrm{H}_{2}^{+}$cyclotron is that ions of the same charge-to-mass ratios can also be accelerated. Specifically, $\mathrm{He}^{++}$(alpha-particle) beams can be accelerated at currents limited only by the availability of such $\mathrm{He}^{++}$ion sources. There are many isotopes that have tremendous application potential and are limited today only by the very restricted availability of suitable high-current alpha beams. In fact, the first prototype cyclotron to be built for testing injection of the high-current $\mathrm{H}_{2}^{+}$beams, to be built at the LNS in Catania, Italy, is being designed to be used directly following the $\mathrm{H}_{2}^{+}$injection tests as a dedicated alphaparticle cyclotron for producing radiotherapeutic isotopes. One example will be ${ }^{211} \mathrm{At}$, which is in short supply for even long term clinical studies 
TABLE 1: Medical isotopes relevant to IsoDAR energies, reprinted from [18].

\begin{tabular}{lcl}
\hline Isotope & Half-life & Use \\
\hline${ }^{52} \mathrm{Fe}$ & $8.3 \mathrm{~h}$ & $\begin{array}{l}\text { The parent of the PET isotope }{ }^{52} \mathrm{Mn} \text { and } \\
\text { iron tracer for red-blood-cell formation } \\
\text { and brain uptake studies. }\end{array}$ \\
\hline${ }^{122} \mathrm{Xe}$ & $20.1 \mathrm{~h}$ & $\begin{array}{l}\text { The parent of PET isotope }{ }^{122} \mathrm{I} \text { used to } \\
\text { study brain blood flow. }\end{array}$ \\
\hline${ }^{28} \mathrm{Mg}$ & $21 \mathrm{~h}$ & $\begin{array}{l}\text { A tracer that can be used for bone studies, } \\
\text { analogous to calcium }\end{array}$ \\
\hline${ }^{128} \mathrm{Ba}$ & $2.43 \mathrm{~d}$ & $\begin{array}{l}\text { The parent of positron emitter }{ }^{128} \mathrm{Cs} . \\
\text { As a potassium analog, this is used for } \\
\text { heart and blood-flow imaging. }\end{array}$ \\
\hline${ }^{97} \mathrm{Ru}$ & $2.79 \mathrm{~d}$ & $\begin{array}{l}\text { A } \gamma \text {-emitter used for spinal fluid and liver } \\
\text { studies. }\end{array}$ \\
\hline${ }^{117 \mathrm{~m}} \mathrm{Sn}$ & $13.6 \mathrm{~d}$ & $\begin{array}{l}\text { A } \gamma \text {-emitter potentially useful for bone } \\
\text { studies. }\end{array}$ \\
\hline & The parent of positron emitter ${ }^{82} \mathrm{Rb}$, a \\
potassium analogue \\
${ }^{82} \mathrm{Sr}$
\end{tabular}

The DAE $\delta$ ALUS superconducting ring cyclotron, in extending the performance of today's record-holding PSI by increasing energy from 590 to $800 \mathrm{MeV}$ and a factor of five in current, becomes a member of the GeV-10 MWclass of machines. Many such machines have been designed and proposed but cost has been an impediment to their realization. To date, only one such project has progressed to the advanced R\&D and construction phase: MYRRHA [73] to be sited in Mol, Belgium. These projects all fall within the Accelerator-Driven Systems (ADS) category, such as nuclear waste transmutation, driving of subcritical thorium-based reactors, and tritium production.

Along with the physics possibilities previously described, the DAE $\delta$ ALUS cyclotrons provide new opportunities in this field by offering beams at a substantially reduced cost over the linear accelerators which until now have been viewed as the only viable technology to reach these levels of beam power in the $\mathrm{GeV}$ energy range. With successful development of these cyclotrons, a substantial growth in the ADS field can be anticipated, with the cost hurdle being surpassed.

3.5. R\&D Status and Plans for the Program. We are employing a four-phase, step-wise approach for the development of the components of the DAE $\delta$ ALUS cyclotrons. While no single component must operate far beyond existing technology, when the smaller steps are combined, the result is a substantial leap forward.

Phase I involves development and testing of an ion source and low-energy beam transport system, including design of the inflection system that guides the beam into the cyclotron. Phase II establishes the injector cyclotron, which is used, with relatively small modifications, for IsoDAR. Phase III will produce the DSRC and associated target/dump, which represent the first full accelerator module. This module can be used for near-accelerator physics with an ultralarge detector for short baseline Beyond Standard Model Searches [74, 75]. Phase IV introduces the necessary design modifications for high-power running needed at the mid and far sites for $\mathrm{CP}$ violation studies.

Of the four phases, phase I is most advanced. This study

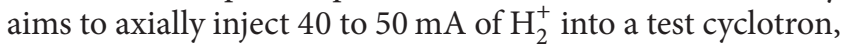
with $5 \mathrm{~mA}$ captured and accelerated to few $\mathrm{MeV}$ energies. As the first step, we have installed a $\sim 15 \mathrm{~mA} \mathrm{H}_{2}^{+}$ion source at the test stand at Best Cyclotron Systems (BCS), Inc. This allows us to gain information for simulating the final spiral inflector. The inflector is unusually large to account for the high beam current and large beam size which must be inflected from the vertical to the horizontal plane. The gap between the electrodes is $15 \mathrm{~mm}$, instead of the typical 6 to $10 \mathrm{~mm}$ gaps found in most cyclotrons. As a result of the size, the spiral inflector occupies a region where the variation of the magnetic field is not negligible, as is assumed in the analytical treatment of spiral inflector design. This effect must be carefully taken into account in the shaping of the electrodes. The design must also minimize the introduction of a high energy spread during the beam transport through the device. Beam neutralization and image charge effects add to the difficulties in producing a reliable design on the first iteration. Our approach has been to take the simulations as far as possible, producing a first spiral inflector, which we are now running at BCS. From this, we are obtaining experimental measurements which will allow us to adjust the design. This will inform our second iteration test stand, which will be built at INFN in Catania, Italy.

An interesting technical challenge that is unique to the $\mathrm{CP}$ program, and not to IsoDAR, is the removal of ions in the high vibrational states from the $\mathrm{H}_{2}^{+}$beam. At $800 \mathrm{MeV}$, in the $6 \mathrm{~T}$ field outer field of the DSRC, the high vibrational states will Lorentz-strip, producing as much as $10 \%$ beam loss. Calculations show that the lowest four states will be stable [76] and so we are investigating ways to remove vibrational states above these. Work in collaboration with Oak Ridge National Laboratory retested the methods of Sen [77], which involve introducing a noble gas into the ion source. Results were difficult to interpret in the first round of tests; however, dissociation by this mechanism is believed to require long (millisecond) residency times of the ions in the source. If so, then we must consider a redesign of the source [77] or a method of removing the vibrational states after the $\mathrm{H}_{2}^{+}$exits the source. Alternative sources are now under discussion.

Phase II is also underway. This crucial step, which is the construction of an IsoDAR source described in the following section, produces a high-current cyclotron very similar to the DAE $\delta$ ALUS injector, at which we extract $5 \mathrm{~mA}$ of $\mathrm{H}_{2}^{+}$ (or $10 \mathrm{~mA}$ of protons) at $60 \mathrm{MeV} / \mathrm{amu}$. As discussed above, success of these cyclotrons depends largely on control of beam "blowup" from space charge. The difficulty of the problem can be gauged using the benchmark of generalized perveance, given in (2), to compare space-charge effects to existing machines. The $5 \mathrm{~mA} \mathrm{H}_{2}^{+}$beam injected into the cyclotron at $70 \mathrm{keV}(35 \mathrm{keV} / \mathrm{amu})$ results in a $K$ value similar to that of existing cyclotrons that inject $2 \mathrm{~mA}$ of protons at $30 \mathrm{keV}$. Thus, while space charge issues are a challenge, we have a reason to expect that the problems can be solved. 
The primary issue for phase III development is the DRSC. Our plans for this have been described above. The $1 \mathrm{MW}$ target is part of the phase III development. Although our machines are designed to operate at higher power, we can limit the average power on target to $1 \mathrm{MW}$. This is done through extracting via multiple stripping foils to separate dumps. Note that the beam will be painted over a $30 \mathrm{~cm}$ target face, greatly reducing power issues.

Phase IV, which is at the least advanced stage, takes phase III system to high power. The collaboration has several competing conceptual designs on how to achieve this. One involves interleaving beam from two injector cyclotrons, while another involves injecting two beams into the central region.

\section{IsoDAR}

IsoDAR is phase II step of DAE $\delta$ ALUS. IsoDAR represents both a novel concept of application to neutrino physics measurements and a demonstration of the $60 \mathrm{MeV} / \mathrm{n}$ injector cyclotron relevant to the larger program.

The baseline cyclotron design for IsoDAR is a $5 \mathrm{~mA} \mathrm{H}_{2}^{+}$ machine that will accelerate beam to $60 \mathrm{MeV}$ per nucleon. Beam would be injected at $70 \mathrm{keV}(35 \mathrm{keV} / \mathrm{amu})$ via a spiral inflector. The cyclotron design used for IsoDAR is very similar to that used for the DIC.

The current plan for IsoDAR is to locate a cyclotron accelerator underground in an experimental hall close to the KamLAND detector, in Kamioka, Japan. This is a continuouswave source with a $90 \%$ duty cycle to allow for machine maintenance. The resulting beam will be transported for a short distance up the drift at KamLAND to a target located in a room. The end of the beam dump is assumed to be $16 \mathrm{~m}$ from the detector center.

We continue to optimize the target for the production of ${ }^{8} \mathrm{Li}$, a $\beta$-emitter, and the source of the $\bar{\nu}_{e}$ for the measurement. The baseline design for the target is a cylinder of ${ }^{9} \mathrm{Be}, 20 \mathrm{~cm}$ long and $20 \mathrm{~cm}$ in diameter. This cylinder is surrounded by an additional $5 \mathrm{~cm}$ of $\mathrm{D}_{2} \mathrm{O}$ which works to both moderate neutrons and provide target cooling. The $\mathrm{D}_{2} \mathrm{O}$ is then surrounded by a cylindrical sleeve of $99.99 \%$ pure ${ }^{7} \mathrm{Li}, 150 \mathrm{~cm}$ long and $200 \mathrm{~cm}$ in outer diameter. Some ${ }^{8} \mathrm{Li}$ is produced directly in the ${ }^{9} \mathrm{Be}$ target but the majority of the ${ }^{8} \mathrm{Li}$ is produced by many neutrons made in the ${ }^{9} \mathrm{Be}$ target capturing on ${ }^{7} \mathrm{Li}$. The isotopic purity of the ${ }^{7} \mathrm{Li}$ sleeve is needed to avoid production of tritium by neutrons on ${ }^{6} \mathrm{Li}$. Further, this production cross-section is several orders of magnitude larger than neutron capture on ${ }^{7} \mathrm{Li}$ and therefore reduces the production of ${ }^{8} \mathrm{Li}$ severely. The needed level of ${ }^{7} \mathrm{Li}$ purity for the sleeve is available as it is commonly used in the nuclear industry. A nominal running period of five years with a $90 \%$ duty cycle produces $1.29 \times 10^{23}$ antineutrinos from the decay of ${ }^{8} \mathrm{Li}$.

When paired with a liquid scintillator detector, this isotope DAR flux opens a number of opportunities for precision neutrino measurements. This paper presents two examples. The first is a high sensitivity sterile neutrino search. The second is a search for new physics in the neutrino sector from neutrino electron scattering. Both cases describe pairing with KamLAND, to provide specific information on rates and backgrounds. An example involving the detection of coherent neutrino scattering is also provided although such a measurement would require a dark-matter-style detector sensitive to $\mathrm{keV}$-scale excitations.

\subsection{Connection between the IsoDAR and DAESALUS Injector} Cyclotrons. While the IsoDAR cyclotron has the same magnetic circuit of the DIC, there are some differences in the acceleration system and the central region in order to improve the high intensity beam production to the level required of the physics we discuss below. An important difference between the DIC and the IsoDAR machine is the duty cycle. The IsoDAR cyclotron will work in CW mode, while the duty cycle of DIC is only $20 \%$, with the modulation on the millisecond scale. The resulting higher beam power of IsoDAR poses a very strong constraint on the fractional beam losses.

The IsoDAR cyclotron will most likely be installed underground, while the DIC cyclotrons can be built at sites on the surface. Of the experimental sites under discussion, KamLAND, at the Kamioka mine in Japan, has particularly narrow access. The horizontal and vertical aperture sizes are $2.4 \mathrm{~m}$ and $3.2 \mathrm{~m}$, respectively, so both transport and assembly pose critical constraints to the cyclotron design. All the machine components must be limited in size and weight, but the machine features like optimum vacuum of $10^{-6} \mathrm{~Pa}$ must be maintained. A plan has been developed by the INFNCatania group for cutting the elements into pieces. While this step sounds drastic, the TRIUMF $500 \mathrm{MeV}$ cyclotron is an existence proof that cyclotrons can be sliced.

4.2. Sterile Neutrino Searches. Searches for light sterile neutrinos with mass $\sim 1 \mathrm{eV}$ are motivated by observed anomalies in several experiments. Intriguingly, these results come from a wide range of experiments covering neutrinos, antineutrinos, different flavors, and different energies. Short baseline accelerator neutrino oscillation experiments $[61,78]$, short baseline reactor experiments $[20,79]$, and even the radioactive source experiments, which were originally intended as calibrations for the chemical solar neutrino experiments [4, 5], have all observed anomalies that can be interpreted to be due to one or more sterile neutrinos.

To understand these anomalies in terms of the $\nu \mathrm{SM}$ for neutrino oscillations, the observations must be compared to the data from the large range of experiments with null results $[62,80-82]$ and then to a model. These "global fits" are most often to models with one or more sterile neutrinos added to the oscillation probability calculation [83]. The extended models are referred to as " $3+1$ ", " $3+2$ ", or " $3+3$ " neutrino models depending on the number of additional sterile neutrinos. The global fits tend to prefer $3+2$ and $3+3$ models with CP violation; $3+1$ models are very hard to reconcile between the experiments with signals and those with null results [38].

The diversity of experiments showing these anomalous results has motivated a number of proposals to address them. 


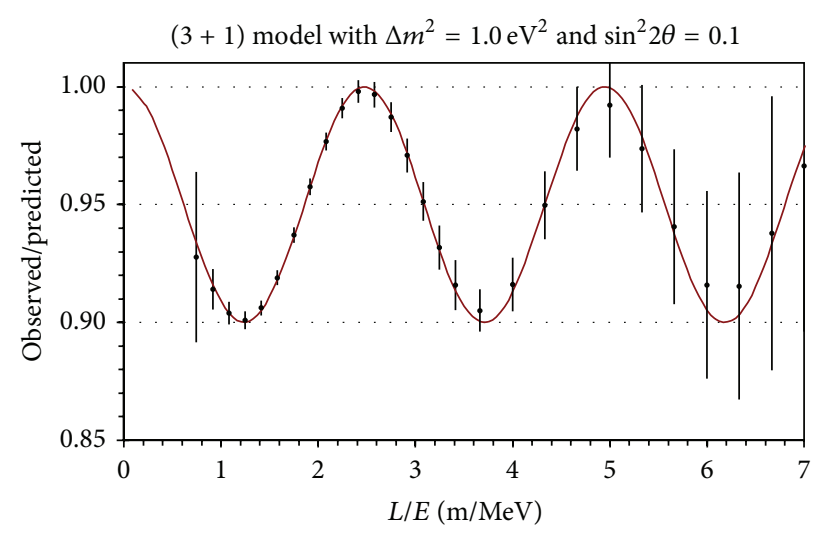

(a)

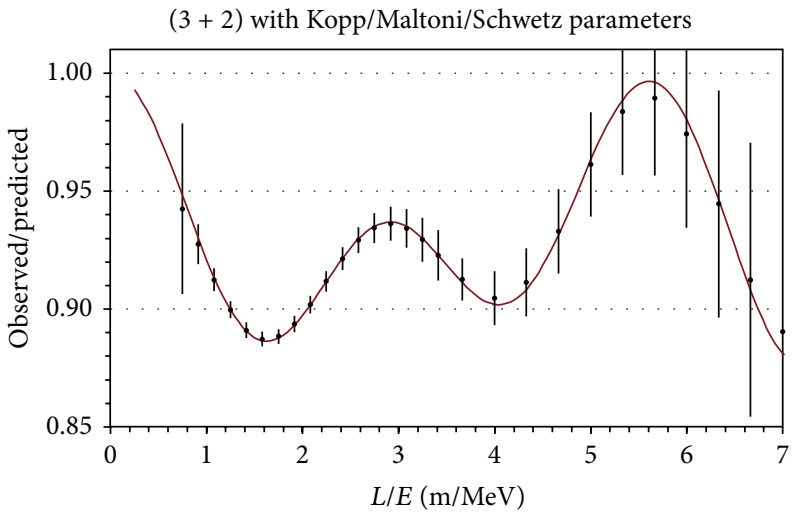

(b)

FIgURE 7: Example data sets for 5 years of running for $3+1$ (a) and $3+2$ (b) oscillation scenarios.

Suggestions range from repeating the source experiments, to specially designed reactor antineutrino experiments, to accelerator-based experiments. Many of these proposals, however, do not have sufficient sensitivity to make a definitive $>5 \sigma$ statement about the existence of sterile neutrinos in all of the relevant parameter space. The experiments that are designed to make a definitive measurement are based on pion or isotope DAR sources. Notably, the full DAE $\delta$ ALUS complex could be used to generate a pion DAR beam for such a measurement. However, the IsoDAR concept calls simply for the DAE $\delta$ ALUS injector cyclotron to be used to generate an isotope DAR source. Such a complex situated next to a kiloton-scale scintillator detector such as KamLAND would enable a definitive search for sterile neutrinos by observing a deficit of antineutrinos as a function of the distance $L$ and antineutrino energy $E$ across the detector-the definitive signature of neutrino oscillation. This is the concept behind the IsoDAR proposal [84].

The proposed IsoDAR target is to be placed adjacent to the KamLAND detector. The antineutrinos propagate $9.5 \mathrm{~m}$ through a combination of rock, outer muon veto, and buffer liquid to the active scintillator volume of KamLAND. The scintillator is contained in a nylon balloon $6.5 \mathrm{~m}$ in radius bringing the total distance from target to detector center to $16 \mathrm{~m}$. The antineutrinos are then detected via the IBD interaction. This interaction has a well known cross-section with an uncertainty of $0.2 \%$ [85] and creates a distinctive coincidence signal between a prompt positron signal, $E_{e^{+}}=$ $E_{\bar{v}_{e}}-0.78 \mathrm{MeV}$, and a delayed neutron capture giving a $2.2 \mathrm{MeV}$ gamma ray within $\sim 200 \mu \mathrm{s}$.

KamLAND was designed to efficiently detect IBD. A standard analysis has a $92 \%$ efficiency for identifying IBD events [86]. In IsoDAR's nominal 5-year run, $8.2 \times 10^{5} \mathrm{IBD}$ events are expected. The largest background comes from the 100 reactor antineutrino IBD events detected by KamLAND per year [87]. The reactor antineutrino rate is dependent on the operation of the nuclear reactors in Japan which has been significantly lower in 2012 and 2013 [88]. The sterile neutrino analysis uses an energy threshold of $3 \mathrm{MeV}$. Due to the effective background rejection efficiency provided by the IBD delayed coincidence signal, this threshold enables use of
TABLE 2: The KamLAND detector parameters used in calculating the sterile neutrino search sensitivity.

\begin{tabular}{lc}
\hline Parameter & Value \\
\hline Run period & 5 years $(4.5$ years live time $)$ \\
$\bar{v}_{e}$ flux & $1.29 \times 10^{23} \bar{\nu}_{e}$ \\
Fiducial mass & 897 tons \\
Target face to detector center & $16 \mathrm{~m}$ \\
Detection efficiency & $92 \%$ \\
Vertex resolutions & $12 \mathrm{~cm} / \sqrt{E(\mathrm{MeV})}$ \\
Energy resolutions & $6.4 \% / \sqrt{E(\mathrm{MeV})}$ \\
Prompt energy threshold & $3 \mathrm{MeV}$ \\
IBD event total & $8.2 \times 10^{5}$ \\
\hline
\end{tabular}

the full KamLAND fiducial volume, $R<6.5 \mathrm{~m}$, and 897 tons, with negligible backgrounds from sources other than from the aforementioned reactor antineutrinos.

The sterile neutrino analysis makes use of neutrino oscillations $L / E$ signature. Therefore, the energy and vertex resolutions are essential in determining sensitivity. The KamLAND detector has a vertex reconstruction resolution of $12 \mathrm{~cm} / \sqrt{E(\mathrm{MeV})}$ and an energy resolution of $6.4 \% / \sqrt{E(\mathrm{MeV})}$ [86]. Example data sets for reasonable 3 +1 and $3+2$ sterile models are shown in Figure 7 for the nominal detector parameters, summarized in Table 2. In most currently favored oscillation scenarios, the $L / E$ signal is observable. Furthermore, separation of the various $3+N$ models may be possible as exemplified by Figure 7(b).

To understand the sensitivity relative to other proposals, the IsoDAR 95\% CL is compared to other electron antineutrino disappearance experiments in the two-neutrino oscillation parameter space in Figure 8. In just five years of running, IsoDAR rules out the entire global $3+1$ allowed region; $\sin ^{2} 2 \theta_{\text {new }}=0.067$ and $\Delta m^{2}=1 \mathrm{eV}^{2}$ at $20 \sigma$. This is the most definitive measurement among the proposals in the most probable parameter space of $\Delta m^{2}$ between $1-10 \mathrm{eV}^{2}$.

4.3. Precision Electroweak Tests of the Standard Model. In addition to the $8.2 \times 10^{5} \mathrm{IBD}$ interactions, the IsoDAR 


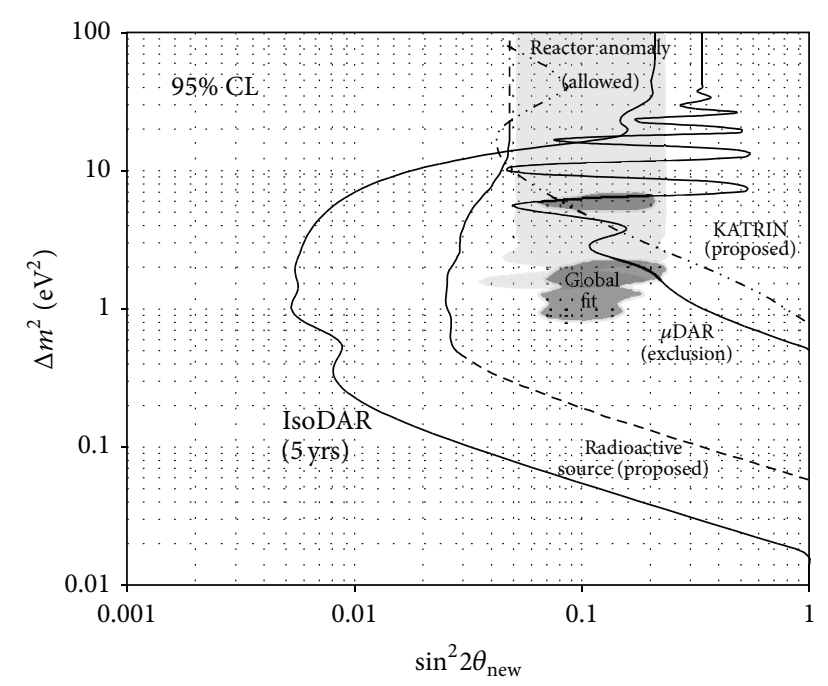

FIGURE 8: Sensitivity of IsoDAR in a nominal 5-year run, in comparison with other experiments. The existing $\mu \mathrm{DAR}[19]$ exclusion curve and Reactor + Gallium [20] allowed region are also shown. With respect to future experiments, the expected sensitivities from a propose PetaBequerel source experiment [21] and from KATRIN [22], now under construction, are also indicated. Reprinted from [23].

neutrino source [23], when combined with the KamLAND detector [89], can collect the largest sample of low-energy $\bar{v}_{e^{-}}$ electron (ES) scatters that has been observed to date. More than 7200 ES events will be collected above a $3 \mathrm{MeV}$ visible energy threshold over a 5-year run, and both the total rate and the visible energy can be measured. This can be compared to the samples from the Irvine experiment (458 events from 1.5 to $3 \mathrm{MeV}$ [90]); TEXONO (414 events from 3 to $8 \mathrm{MeV}$ [91]); Rovno (41 events from 0.6 to $2 \mathrm{MeV}$ [92]); and MUNU (68 events from 0.7 to $2 \mathrm{MeV}$ [93]).

In the Standard Model, the ES differential cross-section is given by

$$
\frac{d \sigma}{d T}=\frac{2 G_{F}^{2} m_{e}}{\pi}\left[g_{R}^{2}+g_{L}^{2}\left(1-\frac{T}{E_{\nu}}\right)^{2}-g_{R} g_{L} \frac{m_{e} T}{E_{\nu}^{2}}\right],
$$

where $T \in\left[0,2 E_{v}^{2} /\left(m_{e}+2 E_{v}\right)\right]$ is the electron recoil energy, $E_{v}$ is the energy of the incoming $\bar{\nu}_{e}$, and the weak coupling constants $g_{R}$ and $g_{L}$ are given at tree level by $g_{R}=\sin ^{2} \theta_{W}$ and $g_{L}=1 / 2+\sin ^{2} \theta_{W}$. Equation (3) can also be expressed in terms of the vector and axial weak coupling constants, $g_{V}$ and $g_{A}$, using the relations $g_{R}=(1 / 2)\left(g_{V}-g_{A}\right)$ and $g_{L}=$ $(1 / 2)\left(g_{V}+g_{A}\right)$.

The ES cross-section can be therefore be used as a probe of the weak couplings, $g_{V}$ and $g_{A}$, as well as $\sin ^{2} \theta_{W}$, a fundamental parameter of the Standard Model as described in [94]. Although $\sin ^{2} \theta_{W}$ has been determined to high precision [95], there is a longstanding discrepancy [16] between the value obtained by $e^{+} e^{-}$collider experiments and the value obtained by $\mathrm{NuTeV}$, a precision neutrino-quark scattering experiment [96]. Despite having lower statistics than the NuTeV, IsoDAR would measure $\sin ^{2} \theta_{W}$ using the purely leptonic ES interaction, which does not involve any nuclear dependence. This could therefore shed some light on the value of $\sin ^{2} \theta_{W}$ measured by neutrino scattering experiments.

The ES cross-section is also sensitive to new physics in the neutrino sector arising from nonstandard interactions (NSIs), which are included in the theory via dimension of six, four-fermion effective operators. NSIs give rise to weak coupling corrections and modify the Standard Model ES cross-section given in (3) to

$$
\begin{aligned}
\frac{d \sigma}{d T}=\frac{2 G_{F}^{2} m_{e}}{\pi}[ & \left(\tilde{g}_{R}^{2}+\sum_{\alpha \neq e}\left|\epsilon_{\alpha e}^{e R}\right|^{2}\right)+\left(\widetilde{g}_{L}^{2}+\sum_{\alpha \neq e}\left|\epsilon_{\alpha e}^{e L}\right|^{2}\right) \\
& \times\left(1-\frac{T}{E_{v}}\right)^{2}-\left(\widetilde{g}_{R} \widetilde{g}_{L}+\sum_{\alpha \neq e}\left|\epsilon_{\alpha e}^{e R}\right|\left|\epsilon_{\alpha e}^{e L}\right|\right) \\
& \left.\times m_{e} \frac{T}{E_{v}^{2}}\right]
\end{aligned}
$$

where $\widetilde{g}_{R}=g_{R}+\epsilon_{e e}^{e R}$ and $\widetilde{g}_{L}=g_{L}+\epsilon_{e e}^{e L}$. The NSI parameters $\epsilon_{e \mu}^{e L R}$ and $\epsilon_{e \tau}^{e L R}$ are associated with flavor-changing-neutral currents, whereas $\epsilon_{e e}^{e L R}$ are called nonuniversal parameters. We can estimate IsoDAR's sensitivity to these parameters by fitting (4) to the measured ES cross section, assuming the Standard Model value for $\sin ^{2} \theta_{W}$. In general, lepton flavor violating processes are tightly constrained so we focus only on IsoDAR's sensitivity to the two non-universal parameters $\epsilon_{e e}^{e L R}$.

The ES interaction used for these electroweak tests of the Standard Model is very different than the IBD interaction used for the sterile neutrino search. The IBD signal consists of a delayed coincidence of a positron and a $2.2 \mathrm{MeV}$ neutron capture $\gamma$, whereas the ES signal consists of isolated events in the detector. Another difference is that, at IsoDAR energies, the IBD cross-section is several orders of magnitude larger than the ES cross section. In fact, if just $1 \%$ of IBD events are misidentified as ES events, they will be the single largest background. On the other hand, as was suggested in [97], the IBD signal can also be used to reduce the normalization uncertainty on the ES signal to about $0.7 \%$. A final difference is that the incoming $\bar{\nu}_{e}$ energy for IBD interactions in KamLAND can be inferred from the visible energy on an event-by-event basis, while the incoming $\bar{\nu}_{e}$ energy for ES interactions in KamLAND cannot. Therefore, the differential ES cross-section is measured in visible energy bins corresponding to the kinetic energy of the recoil electron, and the dependence on the incoming $\bar{\nu}_{e}$ energy is integrated out according to the IsoDAR flux.

The backgrounds to the ES signal can be grouped into beam-related backgrounds, which are dominated by misidentified IBD events, and nonbeam backgrounds, arising from solar neutrino interactions, muon spallation, and environmental sources. We adopt a strategy similar to the one outlined in [98] to reduce the nonbeam backgrounds. First, a cosmic muon veto is applied to reduce the background due to radioactive light isotopes produced in muon spallation inside the detector. This reduces the live time by $62.4 \%$. Next, 
TABLE 3: Total signal and background events in KamLAND with $E_{\text {vis }}$ between 3 and $12 \mathrm{MeV}$ given the IsoDAR assumptions in Table 2 and the selection cuts outlined in text.

\begin{tabular}{lc}
\hline & Events \\
\hline Elastic scattering (ES) & 2583.5 \\
IBD Mis-ID Background & 705.3 \\
Nonbeam Background & 2870.0 \\
Total & $\mathbf{6 1 5 8 . 8}$ \\
\hline
\end{tabular}

TABLE 4: Estimated $\sin ^{2} \theta_{W}$ measurement sensitivity for various types of fits to the $E_{\text {vis }}$ distribution. The second column indicates the background reduction factor.

\begin{tabular}{lcccc}
\hline & $\begin{array}{c}\text { Background } \\
\text { factor }\end{array}$ & $\delta \sin ^{2} \theta_{W}$ & $\frac{\delta \sin ^{2} \theta_{W}}{\sin ^{2} \theta_{W}}$ & $\delta \sin ^{2} \theta_{W}^{\text {stat-only }}$ \\
\hline Rate + shape & 1.0 & 0.0076 & $3.2 \%$ & 0.0057 \\
Shape only & 1.0 & 0.0543 & $22.8 \%$ & 0.0395 \\
Rate only & 1.0 & 0.0077 & $3.2 \%$ & 0.0058 \\
Rate + shape & 0.5 & 0.0059 & $2.5 \%$ & 0.0048 \\
Rate + shape & 0.0 & 0.0040 & $1.7 \%$ & 0.0037 \\
\hline
\end{tabular}

a visible energy threshold of $3 \mathrm{MeV}$ is employed to reduce the background from environmental source which pile up at low energies. Finally, a fiducial radius of $5 \mathrm{~m}$ is used to reduce the background from external gamma rays emanating from the rock or stainless steel surrounding the detector. To reduce the beam-related backgrounds, an IBD veto is employed to reject any ES candidate that is within $2 \mathrm{~ms}$ of a subsequent event with visible energy $>1.8 \mathrm{MeV}$ in a $6 \mathrm{~m}$ fiducial radius. The IBD veto is estimated to have an efficiency of $99.75 \% \pm 0.02 \%$, where the uncertainty is assumed to come from the statistical uncertainty on measuring the IBD selection efficiency with $50,000 \mathrm{AmBe}$ calibration source events.

Table 3 shows the expected signal and background event totals assuming a nominal 5-year IsoDAR run with a $90 \%$ duty factor. We assume that the energy spectrum of the nonbeam backgrounds can be measured with 4.5 years of KamLAND data before the IsoDAR source turns on. The energy spectrum of the nonbeam background, misidentified IBD events, can be extracted from beam-on data with a dedicated delayed coincidence selection. Given these assumptions, Table 4 gives the IsoDAR sensitivity to $\sin ^{2} \theta_{W}$ from a combined fit to the rate and spectral "shape" of the differential ES cross section, as well as each individually. Sensitivities are also shown for the case of a 50\% background reduction and for the case of a 100\% background reduction.

To compare the sensitivity of IsoDAR with that of other experiments, the fits to the ES cross-section can also be done in terms of $g_{V}$ and $g_{A}$. Figure 9 shows the IsoDAR $1 \sigma$ contour in the $g_{V}-g_{A}$ plane as well as contours from other experiments. IsoDAR would be the most sensitive $v_{e} e / \bar{v}_{e} e$ experiment to date and could test the consistency of $\nu_{e} e / \bar{v}_{e} e$ couplings with $\nu_{\mu} e / \bar{\nu}_{\mu} e$ couplings.

Finally, we can also estimate IsoDAR's sensitivity to the non-universal NSI parameters $\epsilon_{e e}^{e L}$ and $\epsilon_{e e}^{e R}$, assuming the Standard Model value for $\sin ^{2} \theta_{W}=0.238$. The results are

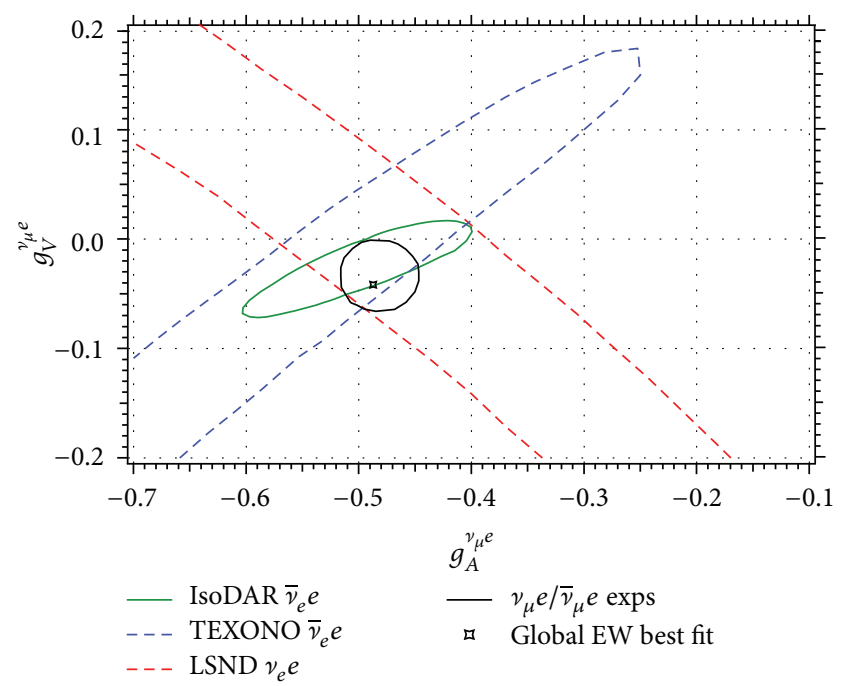

FIGURE 9: IsoDAR's sensitivity to $g_{V}$ and $g_{A}$ along with allowed regions from other neutrino scattering experiments and the electroweak global best fit point taken from [16]. The IsoDAR, LSND, and TEXONO contours are all at $1 \sigma$ and are all plotted in terms of $g_{V, A}^{v_{\mu} e}=g_{V, A}^{v_{e} e}-1$ to compare with $\nu_{\mu}$ scattering data. The $\nu_{\mu} e / \bar{\nu}_{\mu} e$ contour is at $90 \%$ C.L.

shown in Figure 10 along with the current global allowed region [24]. In the region around $\epsilon_{e e}^{e L}$ and $\epsilon_{e e}^{e R} \sim 0$, the IsoDAR $90 \%$ confidence interval significantly improves the global picture.

4.4. Coherent Neutrino Scattering at IsoDAR. As discussed in the previous section, an intense source of neutrinos provides an immense opportunity for a number of physics measurements other than a sterile neutrino search. Along with the weak mixing angle measurement and sensitivity to nonstandard neutrino interactions, such a source could allow the first detection and subsequent high statistics sampling of coherent, neutrino-nucleus scattering events. Although the process is well predicted by the Standard Model and has a comparatively large cross-section in the relevant energy region $(\sim 10-15 \mathrm{MeV})$, neutral current coherent scattering has never been observed before as the low energy nuclear recoil signature is difficult to observe.

A modest sample of a few hundred events collected with a keV-scale-sensitive dark matter style detector could improve upon existing nonstandard neutrino interaction parameter sensitivities by an order of magnitude or more. A deviation from the $\sim 5 \%$ predicted cross-section could be an indication of new physics. Furthermore, the cross-section is relevant to understanding the evolution of core collapse supernovae as well as characterizing future burst supernova neutrino events collected with terrestrial detectors.

A dark matter style detector with $\mathrm{keV}$-scale sensitivity to nuclear recoil events, perhaps based on germanium crystal or single phase liquid argon/neon technology, in combination with an intense proton source such as IsoDAR, could perform the physics discussed above. The technology currently exists for such a detector (with requisite passive and active shielding) to be deployed on the surface or underground. Note that 


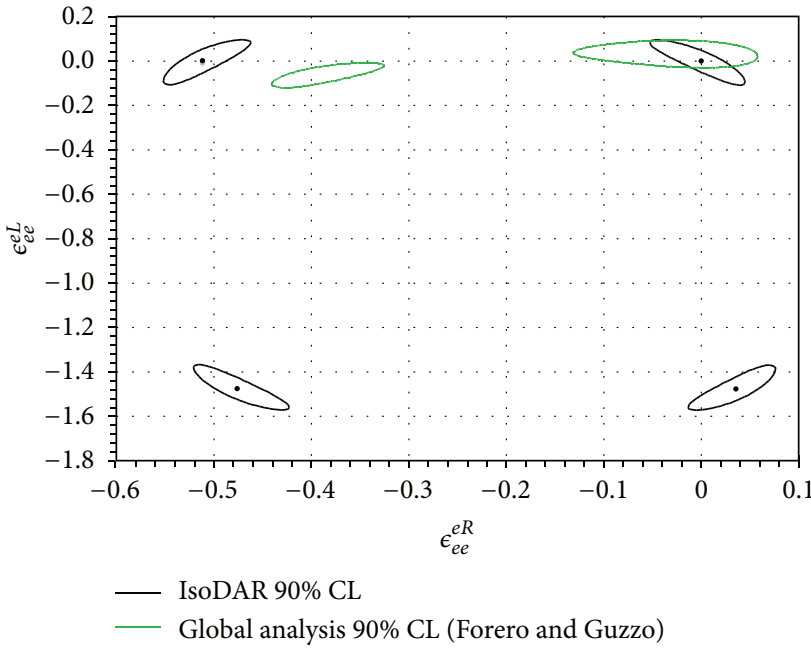

(a)

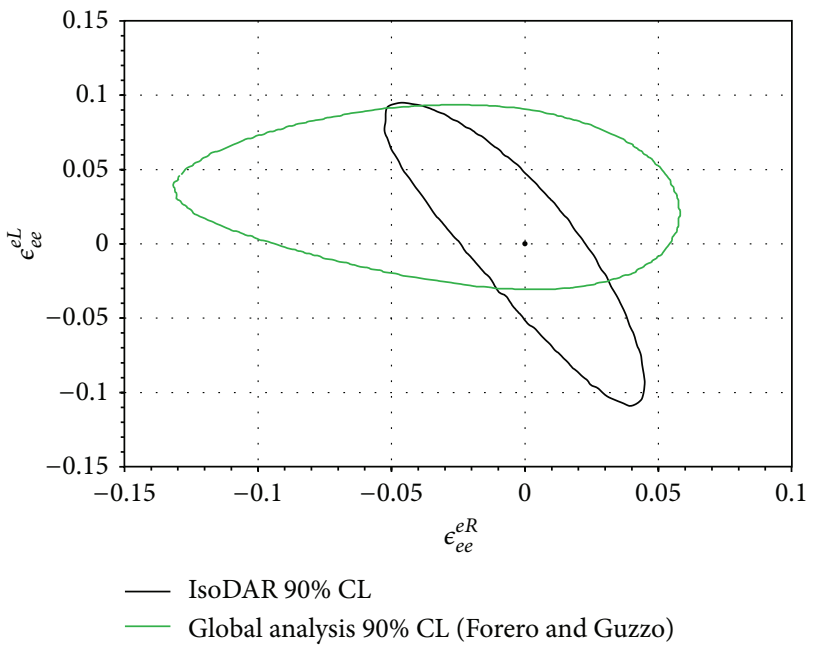

(b)

FIGURE 10: (a) IsoDAR's sensitivity to $\epsilon_{e e}^{e L}$ and $\epsilon_{e e}^{e R}$. The current global allowed region, based on [24], is also shown. (b) A zoomed-in version of the top plot, emphasizing the region near $\epsilon_{e e}^{e L}$ and $\epsilon_{e e}^{e R} \sim 0$, is shown.

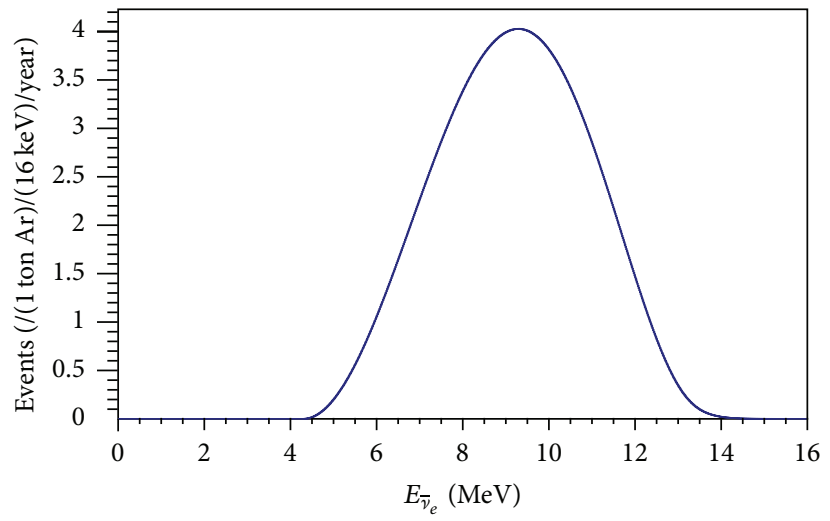

(a)

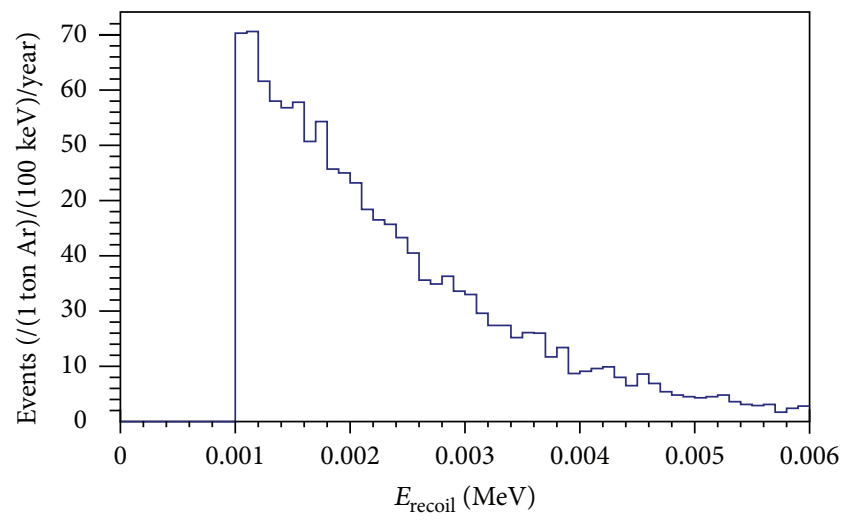

(b)

FIGURE 11: (a) Coherent event rate in terms of antineutrino energy with a $1000 \mathrm{~kg}$ argon detector at a $10 \mathrm{~m}$ average baseline from the IsoDAR source. (b) The event rate in terms of nuclear recoil energy.

the KamLAND detector is not sensitive enough for such a measurement.

Figure 11 shows the expected rates in terms of neutrino energy and nuclear recoil energy for an IsoDAR source $(2.58 \times$ $10^{22} \bar{v}_{e}$ /year) in combination with a $1000 \mathrm{~kg}$ argon detector at a $10 \mathrm{~m}$ average baseline from the source with a $1 \mathrm{keV}$ nuclear recoil energy threshold and 20\% energy resolution. Given these assumptions, about 1200 events per year could be collected for a high statistics sampling of this event class. A first observation of the process is clearly possible with a more modest size detector as well.

\section{DAE $\delta$ ALUS}

5.1. CP Violation Searches. CP violation can occur in neutrino oscillations if there is a complex phase, $\delta_{\mathrm{CP}}$, in the $3 \times 3$ neutrino mixing matrix between the neutrino flavor and mass eigenstates. Observation of CP violation in the light neutrino sector would be a first hint of such effects in the early universe where GUT-scale Majorana neutrinos can have CP violating decays that lead to the matter-antimatter asymmetry that we now observe. This process is called "leptogenesis" [99-101].

The parameter $\delta_{\mathrm{CP}}$ is accessible through the muon-toelectron neutrino flavor oscillation probability. For oscillations in a vacuum, the probability is given by [102]

$$
\begin{aligned}
P_{\mu \rightarrow e}= & \sin ^{2} \theta_{23} \sin ^{2} 2 \theta_{13} \sin ^{2} \Delta_{31} \\
& \mp \sin \delta \sin 2 \theta_{13} \sin 2 \theta_{23} \sin 2 \theta_{12} \sin ^{2} \Delta_{31} \sin \Delta_{21} \\
& +\cos \delta \sin 2 \theta_{13} \sin 2 \theta_{23} \sin 2 \theta_{12} \sin \Delta_{31} \\
& \times \cos \Delta_{31} \sin \Delta_{21}+\cos ^{2} \theta_{23} \sin ^{2} 2 \theta_{12} \sin ^{2} \Delta_{21},
\end{aligned}
$$


where $\Delta_{i j}=\Delta m_{i j}^{2} L / 4 E_{v}$. In the second term, the $-(+)$ refers to neutrino (antineutrino) operation.

A critical parameter for measuring $\mathrm{CP}$ violation is the size of the mixing angle $\theta_{13}$, which determines the size of the first three terms in (5). Recently, several reactor neutrino disappearance experiments (Double Chooz, Daya Bay, and RENO) have made precision measurements of $\theta_{13}$ giving a global average of $\theta_{13}=8.75^{\circ} \pm 0.43^{\circ}$ [45]. The fact that $\theta_{13}$ is now known to be fairly large makes the search for CP violation viable and a key next step in particle physics.

For long baseline experiments, searches for CP violation rely on comparing neutrino and antineutrino oscillation probabilities, thus exploiting the above change of sign in order to isolate $\delta_{\mathrm{CP}}$. This type of measurement is complicated by matter effects, in which the forward scattering amplitude for neutrinos and antineutrinos differs due to the presence of electrons, rather than positrons, in matter.

The matter effects result in a modification of (5) giving

$$
\begin{aligned}
P_{\mu \rightarrow e}^{\text {matter }}= & \sin ^{2} \theta_{23} \sin ^{2} 2 \theta_{13} \frac{\sin ^{2}\left(\Delta_{31} \mp a L\right)}{\left(\Delta_{31} \mp a L\right)^{2}} \Delta_{31}^{2} \\
& \mp \sin \delta \sin 2 \theta_{13} \sin 2 \theta_{23} \sin 2 \theta_{12} \sin \Delta_{31} \\
& \times \frac{\sin \left(\Delta_{31} \mp a L\right)}{\left(\Delta_{31} \mp a L\right)} \Delta_{31} \frac{\sin (a L)}{(a L)} \Delta_{21} \\
& +\cos \delta \sin 2 \theta_{13} \sin 2 \theta_{23} \sin 2 \theta_{12} \cos \Delta_{31} \\
& \times \frac{\sin \left(\Delta_{31} \mp a L\right)}{\left(\Delta_{31} \mp a L\right)} \Delta_{31} \frac{\sin (a L)}{(a L)} \Delta_{21} \\
& +\cos ^{2} \theta_{23} \sin ^{2} 2 \theta_{12} \frac{\sin ^{2}(a L)}{(a L)^{2}} \Delta_{21}^{2} .
\end{aligned}
$$

In this equation, $a=G_{F} N_{e} / \sqrt{2}$ and $\mp$ refer to neutrinos (antineutrinos). Matter effects only appear when $L$ is large, because $a \approx(3500 \mathrm{~km})^{-1}$ (with $\left.\rho Y_{e}=3.0 \mathrm{~g} / \mathrm{cm}^{3}\right)$ is small. Short-baseline experiments such as DAE $\delta$ ALUS have negligible matter effects and moderate baseline experiments such as T2K at $L=295 \mathrm{~km}$ suffer only modest $(\sim \pm 10 \%)$ matter effects. On the other hand, long baseline experiments such as $\mathrm{NO} v \mathrm{~A}$ and LBNE have significant matter effects [44]. The terms that are modified by the matter effects also depend on $\operatorname{sign}\left(\Delta m_{31}^{2}\right)$, making the corrections dependent on knowing this sign, commonly called the "mass hierarchy." Measurement of the mass hierarchy is a goal of several upcoming experiments, including $\mathrm{NO} v \mathrm{~A}$ [103] and PINGU [104]; however it is unclear how well the hierarchy will be known at the time when LBNE will run.

For long baseline accelerator oscillation experiments, gathering sufficient antineutrino data sets is difficult due to the reduced negative pion production rate by accelerator protons and by the reduced interaction cross-section of antineutrinos. The current event estimates for the LBNE experiment with a 34 kton liquid argon detector at $1300 \mathrm{~km}$ from the Fermilab site are shown in Figure 12 [44] for the normal mass hierarchy.
In contrast, the DAE $\delta$ ALUS experiment will be a search only in the antineutrino mode $\bar{\nu}_{\mu} \rightarrow \bar{\nu}_{e}$ with no matter effects, and with reduced backgrounds and systematic uncertainties, plus a unique experimental layout in which several low-cost neutrino sources are at different distances from one large detector. With an antineutrino-only beam, the oscillation probability is given by (5), and the sensitivity to $\mathrm{CP}$ violation comes about through the interference between $\Delta_{12}$ and $\Delta_{13}$ transitions, which have a distinctive $L$ dependence.

Specifically, DAE $\delta$ ALUS will search for $\bar{\nu}_{\mu} \rightarrow \bar{\nu}_{e}$ oscillations using neutrinos from three stopped-pion DAR sources, which interact in a single large 200 to 500 kton Gd-doped water Cherenkov or a large 50 kton liquid scintillator detector. The spectrum of $\bar{\nu}_{\mu}$ 's that can oscillate into $\bar{\nu}_{e}$ 's is shown in Figure 4 . The detection of the electron antineutrinos is done through the IBD process where the outgoing positron is once again required to have a delayed coincidence with a neutron capture on Gd for the water detector or on hydrogen for the scintillator detector. This process has a high cross-section at $\approx 50 \mathrm{MeV}$ but requires $\mathrm{Gd}$ doping for either a water detector or a scintillator detector to detect the outgoing neutron and separate the IBD events from the preponderance of chargedcurrent $\nu_{e}$ events.

The accelerators will be positioned at $1.5,8$, and $20 \mathrm{~km}$ from the large detector as shown in Figure 13, all above ground to reduce the installation and running complexity. This also allows for the proton beam on target to be easily directed somewhat upward, so that any decay-in-flight neutrinos are directed away from the detector. Each accelerator provides different physics data for the $\mathrm{CP}$ violation search. The $1.5 \mathrm{~km}$ accelerator allows measurement of the beam-on backgrounds and the normalization. The $8 \mathrm{~km}$ site is at an oscillation wavelength of about $\pi / 4$ at $50 \mathrm{MeV}$ and the $20 \mathrm{~km}$ site is at oscillation maximum for this energy. Each site will be run for $20 \%$ of the time so that the events from a given source distance can be identified by their time-stamp with respect to this running cycle. This will leave $40 \%$ of the time for beam-off running to measure the nonbeam backgrounds and provide other physics data. The baseline plan is for a tenyear run with $1 \mathrm{MW}, 2 \mathrm{MW}$, and $5 \mathrm{MW}$ neutrino sources at the $1.5,8$, and $20 \mathrm{~km}$ sites, respectively.

Combining the data from the three accelerators helps to minimize the systematic uncertainties associated with the beam and detector and leads to a highly sensitive search for $\mathrm{CP}$ violation. The shape of the DAR flux with energy is known to be of high precision and is common among the various distances; thus shape comparisons will have small uncertainties. The interaction and detector systematic errors are low since all events are detected in a single detector. The fiducial volume error on the IBD events is also small due to the extreme volume-to-surface-area ratio of the ultralarge detector. Therefore, the main errors for the measurements are related to the statistics of the data and to normalization uncertainties. The normalization uncertainties are dominated by the uncertainty of the neutron tagging efficiency, assumed to be $0.5 \%$, and the antineutrino flux uncertainties that are constrained as described next.

The DAE $\delta$ ALUS CP violation analysis follows three steps. First, the absolute normalization of the flux from the near 

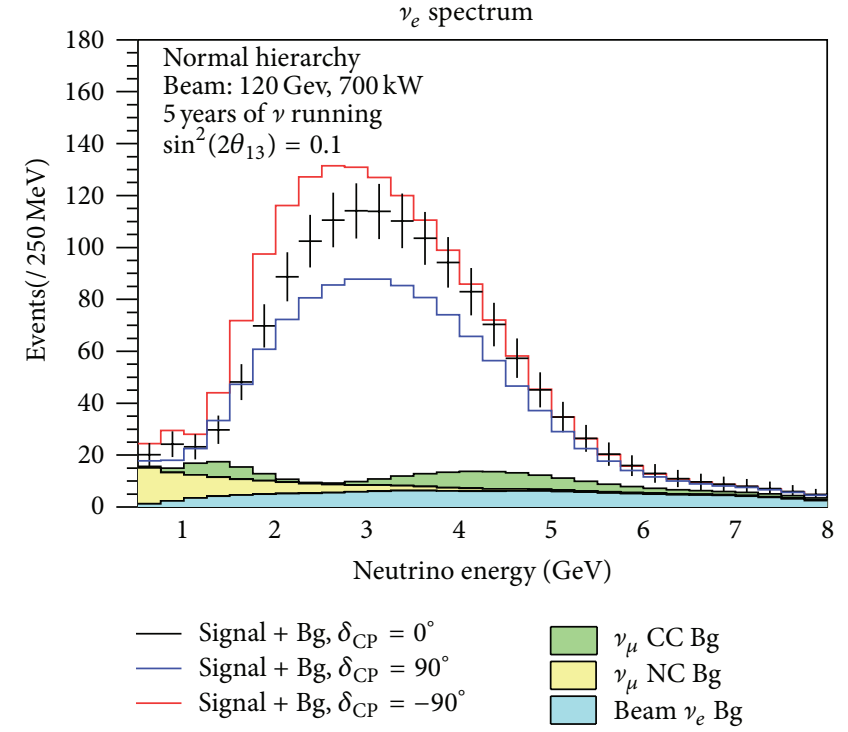

(a)

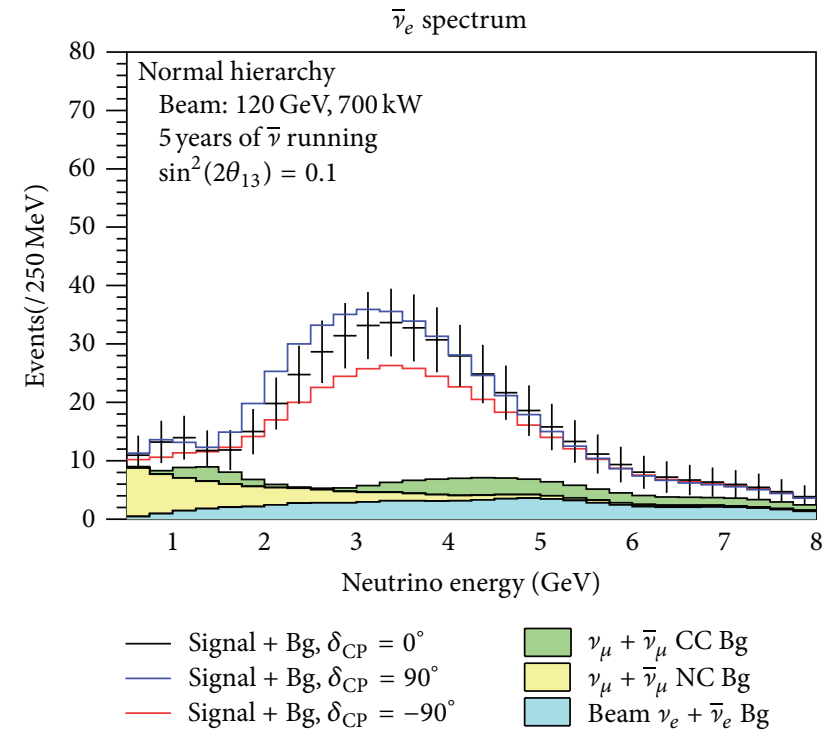

(b)

FIGURE 12: Estimated events for the LBNE experiment running for 5 years of neutrino and 5 years of antineutrino with $700 \mathrm{~kW}$ of $120 \mathrm{GeV}$ protons on target and with the assumption that $\sin ^{2} 2 \theta=0.1$. Backgrounds and changes with $\delta_{\mathrm{CP}}$ are also shown. These plots are from [25].

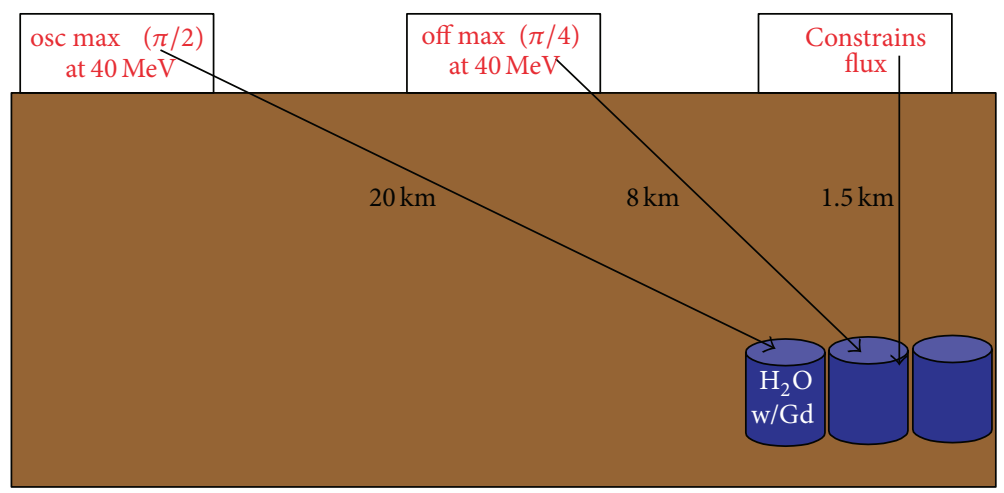

FIGURE 13: Schematic of the DAE $\delta$ ALUS experiment. Three neutrino source locations are used in conjunction with a large water Cherenkov or scintillator-based detector.

accelerator is measured using the $>21,000$ neutrino-electron scatters from that source in the detector, for which the cross-section is known to $1 \%$. The relative flux normalization between the sources is then determined using the comparative rates of charged current $v_{e}$-oxygen (or $v_{e}$-carbon) interactions in the the detector. Since this is a relative measurement, the cross-section uncertainty does not come in but the high statistics is important. Once the normalizations of the accelerators are known, then the IBD data can be fit to extract the $\mathrm{CP}$ violating parameter $\delta_{\mathrm{CP}}$. The fit needs to include all the above systematic uncertainties along with the physics parameter uncertainties. For $\sin ^{2} 2 \theta_{13}, \sin ^{2} \theta_{23}$, and $\Delta m_{\mathrm{atm}}^{2}$, the errors are $0.005,0.01$, and $5.4 \times 10^{-5} \mathrm{eV}^{2}$, respectively.

DAE $\delta$ ALUS must be paired with water or scintillator detectors that have free proton targets. The original case was developed for a $300 \mathrm{kt} \mathrm{Gd}$ doped water detector at Homestake, in coordination with LBNE [105]. Subsequently,
DAE $\delta$ ALUS was incorporated into a program with the

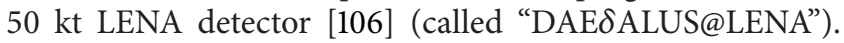
This paper introduces a new study, where DAE $\delta$ ALUS is paired with the Gd-doped $560 \mathrm{kt}$ Hyper-K [26] ("DAESALUS@Hyper-K"). This results in unprecedented sensitivity to CP violation when "DAE $\delta$ ALUS@Hyper-K" data is combined with data from Hyper-K running with the $750 \mathrm{~kW}$ JPARC beam ("DAE $\delta$ ALUS/JPARC@Hyper-K”). In this scenario, JPARC provides a pure $\nu_{\mu}$ flux, rather than running in neutrino and antineutrino mode. This plays to the strength of the JPARC conventional beam, while DAE $\delta$ ALUS provides a high statistics $\bar{\nu}_{\mu}$ flux with no $\nu_{\mu}$ contamination. A summary of the assumptions for the various configuration scenarios is provided in Table 5. CP violation sensitivities have been estimated for 10-year baseline data sets for all the configurations given in Table 5 using a $\Delta \chi^{2}$ fit with pull parameters for each of the systematic uncertainties. For the DAE $\delta$ ALUS configurations, data from all three neutrino 
TABLE 5: Configurations considered in the various $\mathrm{CP}$ violation sensitivity studies.

\begin{tabular}{|c|c|c|c|c|c|}
\hline $\begin{array}{l}\text { Configuration } \\
\text { name }\end{array}$ & Source(s) & $\begin{array}{c}\text { Average long baseline } \\
\text { beam power }\end{array}$ & Detector & Fiducial volume & Run length \\
\hline DAE $\delta$ ALUS@LENA & DAE $\delta$ ALUS only & N/A & LENA & $50 \mathrm{kt}$ & 10 years \\
\hline 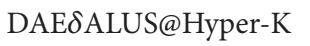 & DAE $\delta$ ALUS only & N/A & Hyper-K & $560 \mathrm{kt}$ & 10 years \\
\hline $\begin{array}{l}\text { DAE } \delta \text { ALUS/JPARC(nu } \\
\text { only)@Hyper-K }\end{array}$ & $\begin{array}{l}\text { DAE } \delta \text { ALUS \& } \\
\text { JPARC }\end{array}$ & $750 \mathrm{~kW}$ & Hyper-K & $560 \mathrm{kt}$ & 10 years \\
\hline JPARC@Hyper-K & JPARC & $750 \mathrm{~kW}$ & Hyper-K & $560 \mathrm{kt}$ & $\begin{array}{c}3 \text { years } \nu+ \\
7 \text { years } \bar{v}[26]\end{array}$ \\
\hline LBNE & FNAL & $850 \mathrm{~kW}$ & LBNE & $35 \mathrm{kt}$ & $\begin{array}{c}5 \text { years } v \\
5 \text { years } \bar{v}[44]\end{array}$ \\
\hline
\end{tabular}

sources are included along with the neutrino electron and $\nu_{e^{-}}$ oxygen (or $\nu_{e}$-carbon) normalization samples. As an example, Table 6 and Figure 14 present a summary of the events by category for the DAE $\delta$ ALUS@Hyper-K configuration. The precision for measuring the $\delta_{\mathrm{CP}}$ parameter in the DAE $\delta$ ALUS@Hyper-K configuration is given in Table 7 for $\sin ^{2} 2 \theta_{13}=0.10$ [45], both for the total and statistical-only uncertainty. The distribution of the uncertainty as a function of $\delta_{\mathrm{CP}}$ is shown in Figure 15. From these estimates, it is clear that, even with the large Hyper-K detector, the measurement is dominated by statistical uncertainty. Also estimates of the measurement uncertainties for the proposed Hyper-K [26] and LBNE [107] experiments for ten-year runs with the proposed upgraded beam intensities $(0.75 \mathrm{MW}$ for HyperK and an average $0.85 \mathrm{MW}$ for LBNE) are shown in the table. Depending on the true value, DAE $\delta$ ALUS has comparable sensitivity for measuring $\delta_{\mathrm{CP}}$ but has very different systematic uncertainties. Thus, DAE $\delta$ ALUS could provide key information that can be used in conjunction with the other experiments to reduce the global measurement uncertainty.

The DAE $\delta$ ALUS high-statistics antineutrino data can be combined with a neutrino-only long baseline measurement to provide improved sensitivity for measuring $\delta_{\mathrm{CP}}$. One possibility is a ten-year neutrino-only run of the JPARC@Hyper-K configuration combined with a ten-year DAESALUS@Hyper-K exposure. The complementarity of the two experiments allows for a very precise search for $\mathrm{CP}$ violation with uncertainties estimated to be around $5^{\circ}$. For this discussion we make the same assumptions that were used for Table 6: a 560 kton Gd-doped water detector, $\sin ^{2} 2 \theta_{13}=$ 0.1 , and $\theta_{23}=49^{\circ}$ [45].

The power of the combined run is shown in Figure 15. Figure 15(a) shows the expectation for the two experiments individually. Nominal JPARC@Hyper-K running assumes three years of running in neutrino mode. This data set would yield the uncertainty indicated by the green diamonds. This would be followed by seven years of running in antineutrino mode. This data set, alone, results in the curve indicated by the green $\times$ symbols. One clearly sees that the strength of JPARC@Hyper-K is in neutrino running, as one would expect from a conventional neutrino beam. Combining these two data sets gives the green solid curve with triangles. DAESALUS@Hyper-K alone, with a 10-year run, results in
TABLE 6: Event samples for the DAE $\delta A L U S @ H y p e r-K$ running scenario for a 10-year run with $\sin ^{2} 2 \theta_{13}=0.1$ [45].

\begin{tabular}{lccc}
\hline Event type & $1.5 \mathrm{~km}$ & $8 \mathrm{~km}$ & $20 \mathrm{~km}$ \\
\hline IBD oscillation events $\left(E_{\mathrm{vis}}>20 \mathrm{MeV}\right)$ & & & \\
$\delta_{\mathrm{CP}}=0^{0}$ & & & \\
$\quad$ Normal hierarchy & 2660 & 4456 & 4417 \\
$\quad$ Inverted hierarchy & 1838 & 3268 & 4338 \\
$\delta_{\mathrm{CP}}=90^{0}$ & & & \\
$\quad$ Normal hierarchy & 2301 & 4322 & 5506 \\
$\quad$ Inverted hierarchy & 2301 & 4328 & 5556 \\
$\delta_{\mathrm{CP}}=180^{0}$ & & & \\
$\quad$ Normal hierarchy & 1838 & 3263 & 4295 \\
$\quad$ Inverted hierarchy & 2660 & 4462 & 4460 \\
$\delta_{\mathrm{CP}}=270^{0}$ & & & \\
$\quad$ Normal hierarchy & 2197 & 3397 & 3206 \\
$\quad$ Inverted hierarchy & 2197 & 3402 & 3242 \\
\hline IBD from intrinsic $\bar{\nu}_{e}\left(E_{\mathrm{vis}}>20 \mathrm{MeV}\right)$ & 1119 & 79 & 31 \\
IBD Nonbeam $\left(E_{\mathrm{vis}}>20 \mathrm{MeV}\right)$ & & & \\
Atmospheric $\nu_{\mu} p$ "invisible muons" & 505 & 505 & 505 \\
Atmospheric IBD & 103 & 103 & 103 \\
Diffuse SN neutrinos & 43 & 43 & 43 \\
\hline$\nu-e$ elastic $\left(E_{\mathrm{vis}}>10 \mathrm{MeV}\right)$ & 40025 & 2813 & 1123 \\
$\nu_{e}-$ oxygen $\left(E_{\mathrm{vis}}>20 \mathrm{MeV}\right)$ & 188939 & 13281 & 5305 \\
\hline
\end{tabular}

TABLE 7: DAE $\delta$ ALUS@Hyper-K $1 \sigma$ measurement uncertainty (in degrees) on $\delta_{\mathrm{CP}}$ for $\sin ^{2} \theta_{13}=0.10$ assuming the baseline 10 -year data sample with a $560 \mathrm{kton}$ Gd-doped water detector. (statisticalonly errors are shown in parentheses). Also an estimate for the JPARC@Hyper-K sensitivity for a 560 kton water detector run for $7.5 \mathrm{MW}$ yrs ( 3 years $\nu$ and 7 years $\bar{\nu}$ ) is shown assuming $5 \%$ systematic errors and the LBNE experiment with a 35 kton liquid argon detector run for $8.5 \mathrm{MW}$ yrs ( 5 years $\nu$ and 5 years $\bar{\nu}$ ).

\begin{tabular}{lccccc}
\hline$\delta_{\mathrm{CP}}$ & $-180^{\circ}$ & $-90^{\circ}$ & $0^{\circ}$ & $90^{\circ}$ & $135^{\circ}$ \\
\hline DAE $\delta$ ALUS@Hyper-K & 9.2 & 12.9 & 10.8 & 18.1 & 16.9 \\
Stat-only & $(8.8)$ & $(11.5)$ & $(10.5)$ & $(15.8)$ & $(16.2)$ \\
JPARC@Hyper-K & 7.8 & 15.2 & 7.8 & 15.0 & 9.1 \\
LBNE & 10.4 & 18.5 & 10.4 & 15.9 & 11.4 \\
\hline
\end{tabular}

the solid red curve. One can see that DAE $\delta$ ALUS@Hyper$\mathrm{K}$ has a similar shape to the JPARC@Hyper-K antineutrino 


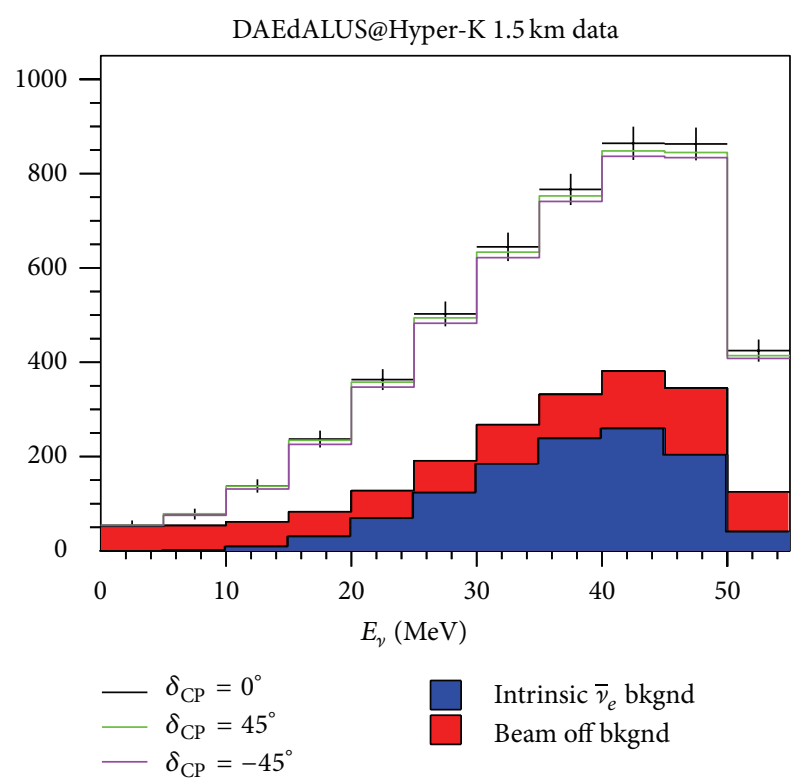

(a)

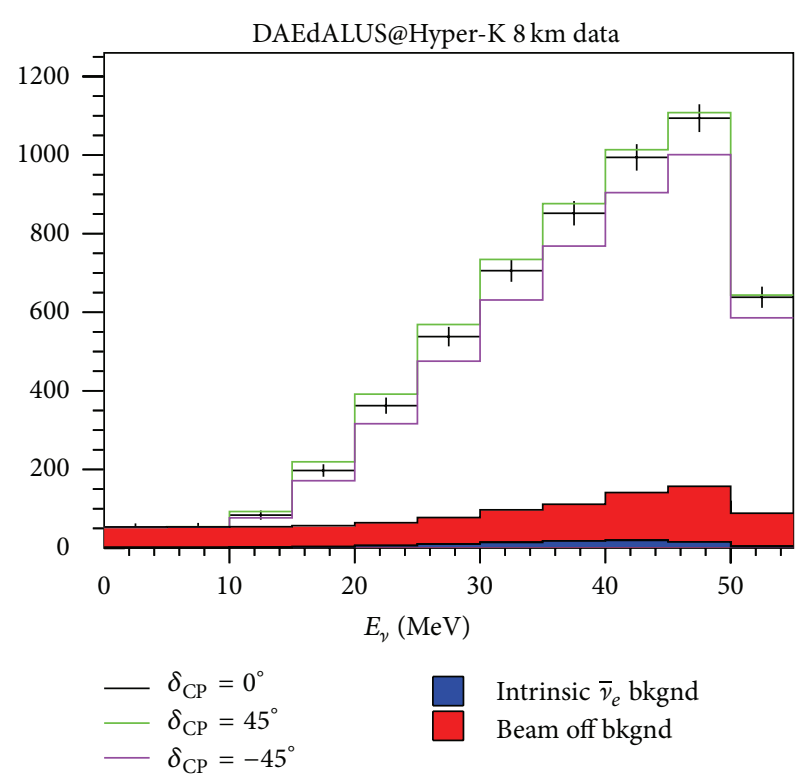

(b)

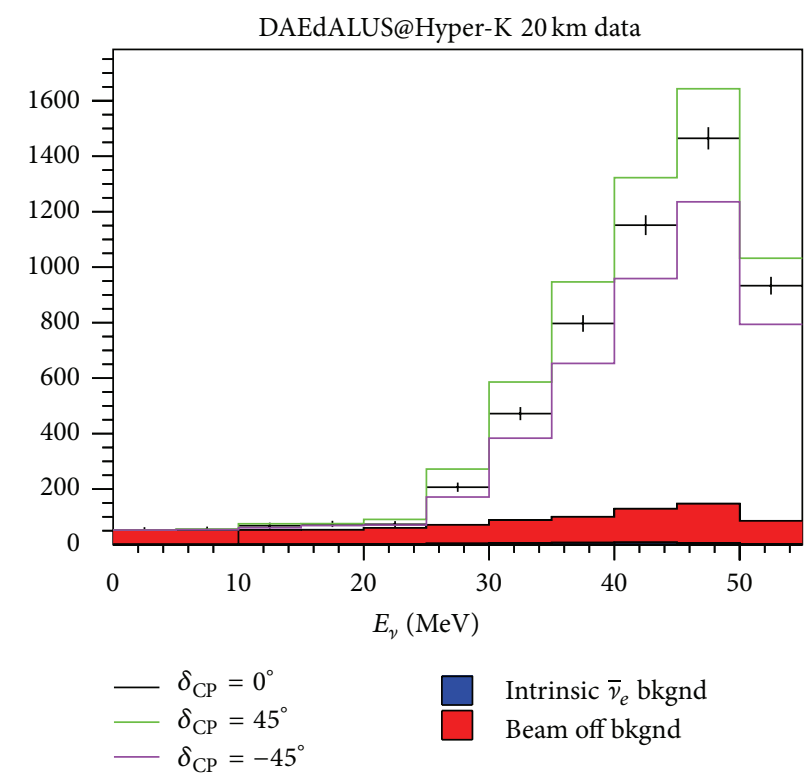

(c)

FIGURE 14: The event energy distributions for signal and background of the DAE $\delta$ ALUS@Hyper-K running scenario with $\sin ^{2} 2 \theta_{13}=0.10$. Black, green, and violet histograms show signals for $\delta_{\mathrm{CP}}=0,45^{\circ}$ and $-45^{\circ}$. The blue histogram shows the intrinsic $\bar{\nu}_{e}$ beam-on background. The red histogram shows the beam-off backgrounds. (a) and (b) show events from the near $(1.5 \mathrm{~km})$ and middle $(8 \mathrm{~km})$ accelerators. (c) shows events from the far $(20 \mathrm{~km})$ accelerator.

running, where the differences come from the additional purity of the $\bar{\nu}_{e}$ flux and the lack of a mass hierarchy effect in the antineutrino data sample from DAE $\delta$ ALUS. We are proposing to combine a JPARC@Hyper-K run in neutrinomode only with the DAE $\delta$ ALUS@Hyper-K pure antineutrino data set. The result is shown on Figure 15(b), by the black curve. The individual contributions of the experiments are also shown. One can see the complementarity, where DAESALUS@Hyper-K provides the strong reach for $\delta_{\mathrm{CP}}<0$ and JPARC@Hyper-K provides the strong reach for $\delta_{\mathrm{CP}}>0$.
Finally, Figure 16 shows the cross-comparison of the experimental configurations shown in Table 5. The combination of DAE $\delta$ ALUS/JPARC(nu-only)@Hyper-K configuration is compared to the two DAE $\delta$ ALUS-only configurations using the LENA and Hyper-K detectors in Figure 16(a) and to JPARC@Hyper-K and LBNE in Figure 16(b). From this figure, it is clear that the DAE $\delta$ ALUS/JPARC(nu-only)@Hyper$\mathrm{K}$ configuration has impressive sensitivity to $\delta_{\mathrm{CP}}$ with a significantly smaller measurement error as compared to any of the other scenarios. Figure 17 shows a comparison of 


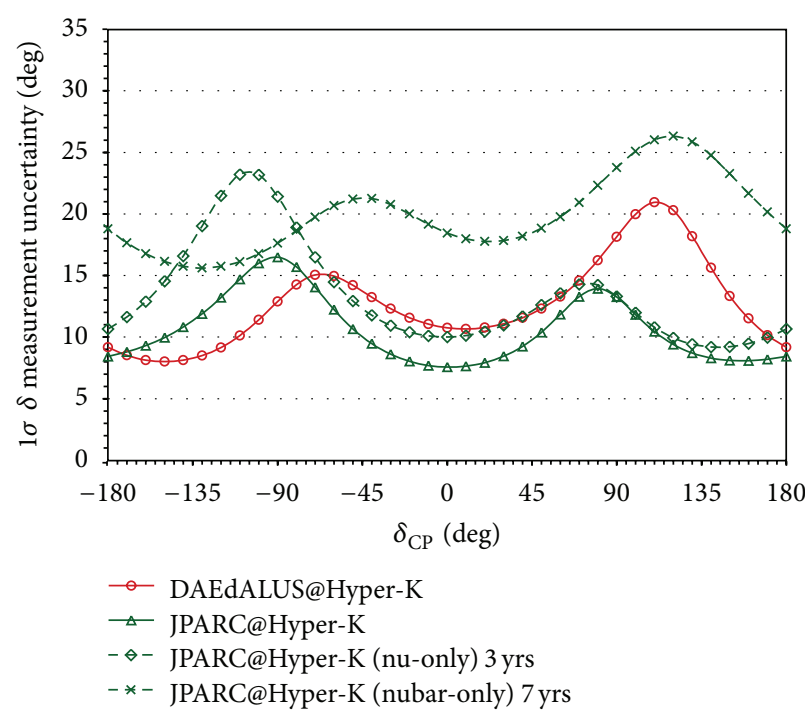

(a)

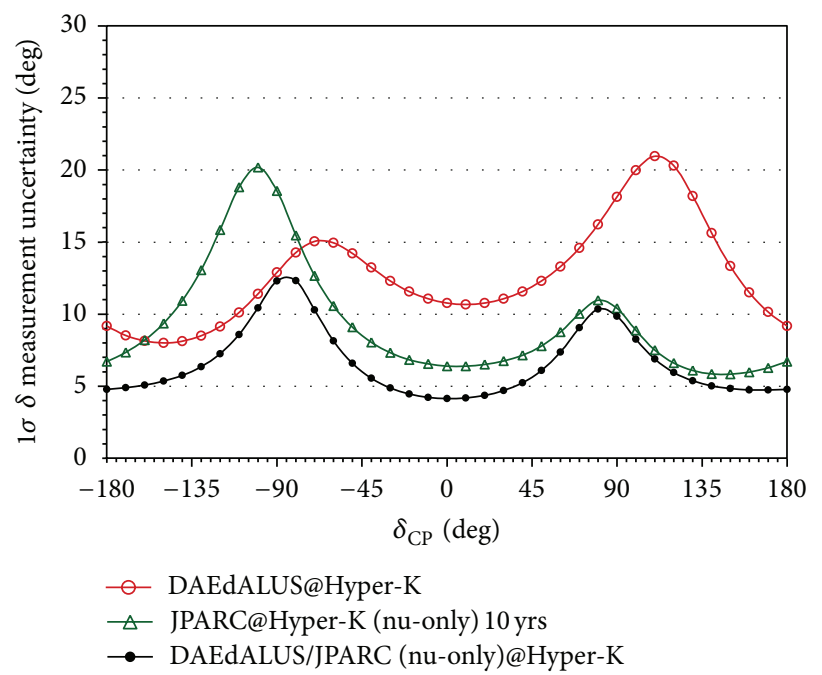

(b)

FIGURE 15: (a) $1 \sigma$ measurement sensitivities for the nominal JPARC@Hyper-K run (solid green with triangles) compared to DAESALUS@Hyper-K (solid red) assuming the normal mass hierarchy. Dashed green curves show contributions of neutrino and antineutrino running to the total sensitivity of JPARC@Hyper-K. (b) DAE $\delta$ ALUS/JPARC(nu only)@Hyper-K combined measurement sensitivity assuming the normal mass hierarchy. The contribution to the combined measurement from DAE $\delta$ ALUS antineutrinos is indicated in red and the contribution from JPARC neutrinos is indicated in green.

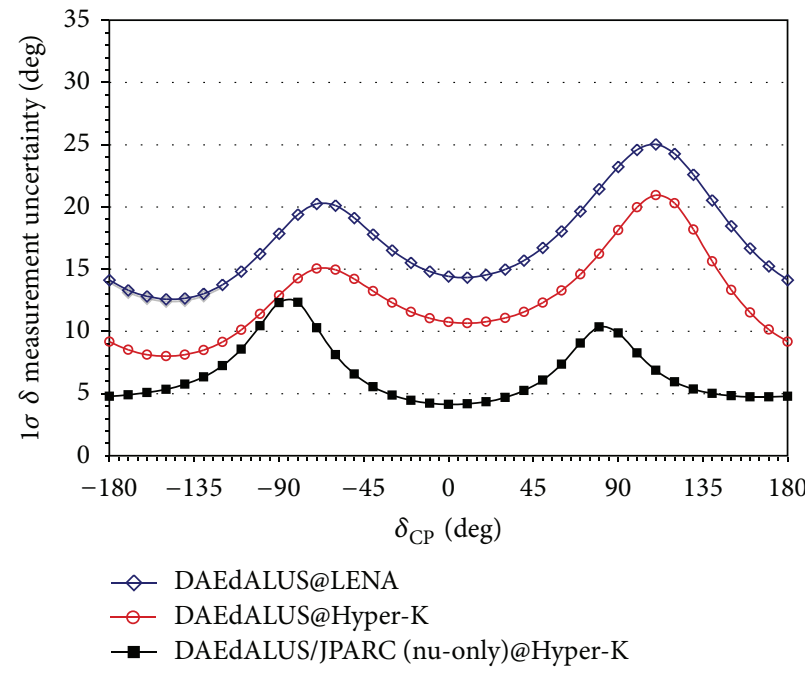

(a)

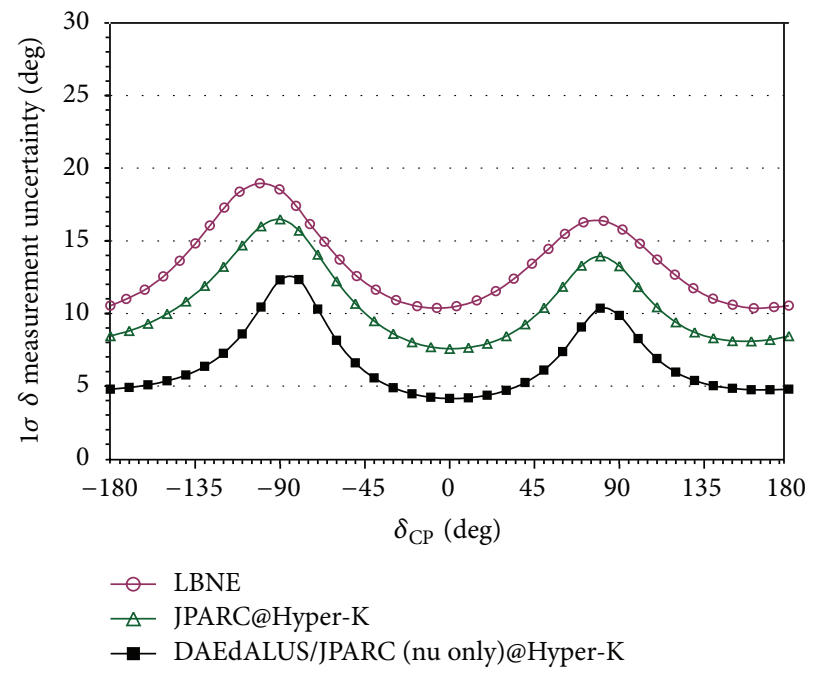

(b)

FIGURE 16: (a) The sensitivity of the CP violation search in various configurations assuming the normal mass hierarchy:

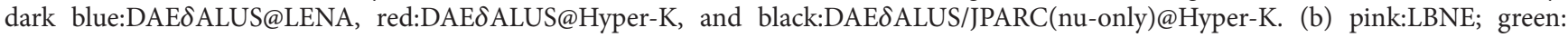

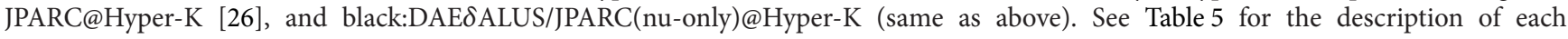
configuration.

the $\delta_{\mathrm{CP}}$ regions where an experiment can discover CP violation by excluding the $\delta_{\mathrm{CP}}=0^{\circ}$ or $180^{\circ}$ at $3 \sigma$ or $5 \sigma$. Again, the DAE $\delta A L U S / J P A R C(n u-o n l y) @ H y p e r-K$ experiment clearly has substantially better coverage.

5.2. Other Physics with DAESALUS. A number of other potential physics opportunities complement the main goal of a measurement of the neutrino $\mathrm{CP}$ violating phase with
DAE $\delta$ ALUS. These experiments can be located near any of the three cyclotron locations. We provide three examples here.

A large water detector, used for a $\sin ^{2} \theta_{W}$ measurement using $v_{e}$-electron scattering [74], would be complementary to the $\bar{\nu}_{e}$-electron search in two ways. First, it explores differences in neutrinos versus antineutrinos that can be introduced by new physics processes. Second, with the ultrahigh 


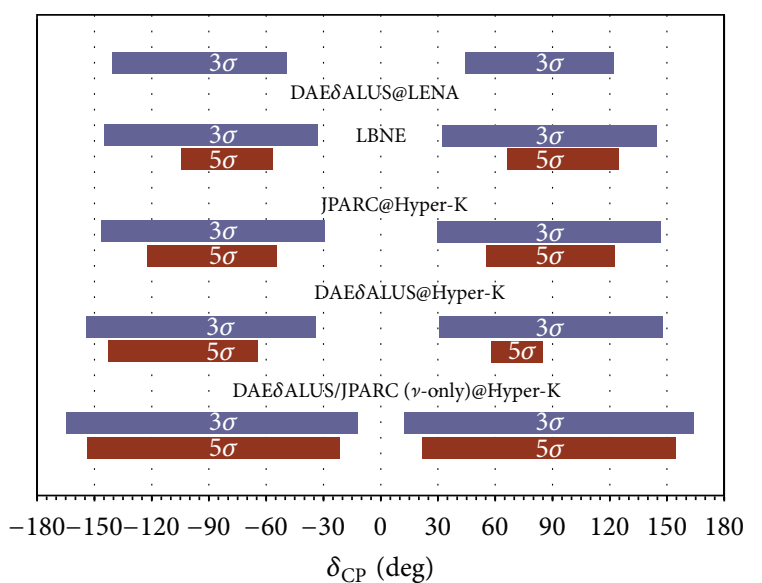

FIGURE 17: The $\delta_{\mathrm{CP}}$ regions where an experiment can discover CP violation by excluding $\delta_{\mathrm{CP}}=0^{\circ}$ or $180^{\circ}$ at $3 \sigma$ or $5 \sigma$. See Table 5 for the description of each configuration.

statistics of the DAE $\delta$ ALUS machines, an energy-dependent analysis, rather than a rate analysis, becomes possible.

A search for $v_{\mu} \rightarrow v_{e}$ appearance at high $\Delta m^{2}$, hence short-baseline, can be performed to address the LSND [61] and MiniBooNE [78] signals if the DAE $\delta$ ALUS configuration uses a large scintillator detector such as LENA [75]. In this case, the cyclotron must be located underground, within tens of meters of the detector. Like the $v_{e}$ disappearance search of IsoDAR, this study searches for the oscillation wave across the detector. Thus it would be powerful confirmation of a sterile-neutrino-related oscillation signal as the source of this high $\Delta m^{2}$ anomaly. The $v_{e}$ flux can also be used for oscillation studies via the disappearance channel [75].

Lastly, a discovery of coherent neutrino scattering is also possible at a DAE $\delta$ ALUS cyclotron. Notably, a cyclotron can provide the source of neutrinos for a coherent discovery at a deep underground detector $1.5 \mathrm{~km}$ away from the source [108] with only a small effect on such a detector's dark matter search exposure. Furthermore, hundreds of coherent events can be collected with a dark-matter-style detector close to such a source for nonstandard neutrino interaction sensitivity. A sensitive, unique neutral-current-based sterile neutrino search using coherent events can also be accomplished [17].

\section{Conclusion}

At the 100th anniversary of Pontecorvo's birth, neutrino physics is entering a new "precision era." To achieve our goals for the next 100 years, improved flux sources are needed. Decay-at-rest sources, driven by cyclotron accelerators, offer neutrino beams of well defined flavor content and with energies in ranges where backgrounds are low and knowledge of the cross-section is high. This paper describes schemes to produce isotope and pion/muon decay-at-rest sources, developed as a part of the DAE $\delta$ ALUS program.

This paper has provided examples of the value of the high precision beams for pursuing new physics. In particular, new results on a combined DAE $\delta$ ALUS-Hyper-K search for $\mathrm{CP}$ violation are presented. This study shows that errors on the mixing matrix parameter ranging from $4 \%$ to $12 \%$ are achievable. While this result is a centerpiece of the program, DAE $\delta$ ALUS and its early phase program, IsoDAR, allow for a wide range of important measurements and searches. Many examples have been presented here, focussing primarily on searches for Beyond Standard Model Physics through oscillations and nonstandard interactions. This establishment of this rich new program is a great way for today's neutrino physicists-the intellectual descendants of Pontecorvo-to celebrate the anniversary of his birth.

\section{Conflict of Interests}

There is no conflict of interests that exists such that the professional judgement of the authors and validity of the research were not influenced by a secondary interest of any kind.

\section{Acknowledgments}

The authors thank the DAE $\delta$ ALUS collaboration for useful discussions. Some studies reported here were initiated at the 2012 Erice International School of Subnuclear Physics Workshop, supported by the Majorana Centre from the INFN Eloisatron Project, which is directed by Professor. Antonino Zichichi. The authors thank the National Science Foundation for support. L. A. Winslow is supported by funds from UCLA.

\section{References}

[1] B. Pontecorvo, "Mesonium and anti-mesonium," Soviet PhysicsJETP, vol. 6, p. 429, 1957.

[2] B. Pontecorvo, "Electron and Muon neutrinos," Soviet PhysicsJETP, vol. 10, pp. 1236-1240, 1960.

[3] R. Davis Jr., D. S. Harmer, and K. C. Hoffman, "Search for neutrinos from the sun," Physical Review Letters, vol. 20, no. 21, pp. 1205-1209, 1968.

[4] J. N. Abdurashitov, V. N. Gavrin, S. V. Girin et al., "Measurement of the solar neutrino capture rate with gallium metal," Physical Review C, vol. 60, no. 5, Article ID 055801, 32 pages, 1999.

[5] W. Hampel, J. Handt, G. Heusser et al., "GALLEX solar neutrino observations: results for GALLEX IV," Physics Letters B, vol. 447, no. 1-2, pp. 127-133, 1999.

[6] K. Hirata, K. Inoue, T. Kajita et al., "Results from one thousand days of real-time, directional solar-neutrino data," Physical Review Letters, vol. 65, no. 11, pp. 1297-1300, 1990.

[7] K. Hirata, T. Kajita, M. Koshiba et al., "Experimental study of the atmospheric neutrino flux," Physics Letters B, vol. 205, no. 2-3, pp. 416-420, 1988.

[8] D. Casper, R. Becker-Szendy, C. B. Bratton et al., "Measurement of atmospheric neutrino composition with the IMB-3 detector," Physical Review Letters, vol. 66, no. 20, pp. 2561-2564, 1991.

[9] W. Allison, G. Alner, D. Ayres et al., “The atmospheric neutrino flavor ratio from a 3.9 fiducial kiloton-year exposure of Soudan 2," Physics Letters B, vol. 449, no. 1-2, pp. 137-144, 1999.

[10] S. Fukuda, Y. Fukuda, M. Ishitsuka et al., "Solar ${ }^{8} B$ and hep neutrino measurements from 1258 days of Super-Kamiokande data," Physical Review Letters, vol. 86, no. 25, pp. 5651-5655, 2001. 
[11] Q. Ahmad, R. C. Allen, T. C. Anderson et al., "Measurement of the rate of $v_{e}+d \rightarrow \rho+\rho+e^{-}$-interactions produced by ${ }^{8} B$ solar neutrinos at the sudbury neutrino observatory," Physical Review Letters, vol. 87, no. 7, Article ID 071301, 6 pages, 2001.

[12] T. Araki, K. Eguchi, S. Enomoto et al., "Measurement of neutrino oscillation with KamLAND: evidence of spectral distortion," Physical Review Letters, vol. 94, no. 8, Article ID 081801, 5 pages, 2005.

[13] M. Ahn, E. Aliu, S. Andringa et al., "Measurement of neutrino oscillation by the K2K experiment," Physical Review D, vol. 74, no. 7, Article ID 072003, 39 pages, 2006.

[14] D. Michael, P. Adamson, and T. Alexopoulos, "Observation of Muon neutrino disappearance with the MINOS detectors in the NuMI neutrino beam," Physical Review Letters, vol. 97, no. 19, Article ID 191801, 6 pages, 2006.

[15] K. Abe, N. Abgrall, Y. Ajima et al., "First muon-neutrino disappearance study with an off-axis beam," Physical Review D, vol. 85, no. 3, Article ID 031103, 2012.

[16] J. Beringer, J. Arguin, R. Barnett et al., "Review of particle physics," Physical Review D, vol. 86, no. 1, Article ID 010001, 1528 pages, 2012.

[17] A. Anderson, J. M. Conrad, E. Figueroa-Feliciano et al., "Measuring active-to-sterile neutrino oscillations with neutral current coherent neutrino-nucleus scattering," Physical Review $D$, vol. 86, no. 1, Article ID 013004, 11 pages, 2012.

[18] A. Adelmann, J. R. Alonso, and W. Barletta, "Costeffective design options for IsoDAR," 2012, http://arxiv.org/ abs/1210.4454.

[19] J. Conrad and M. Shaevitz, "Limits on electron neutrino disappearance from the KARMEN and LSND $v_{e}$-carbon cross section data," Physical Review D, vol. 85, no. 1, Article ID 013017, 6 pages, 2012.

[20] G. Mention, M. Fechner, Th. Lasserre et al., "Reactor antineutrino anomaly," Physical Review D, vol. 83, no. 7, Article ID 073006, 20 pages, 2011.

[21] M. Cribier, M. Fechner, T. Lasserre et al., "Proposed search for a fourth neutrino with a PBq antineutrino source," Physical Review Letters, vol. 107, no. 20, Article ID 201801, 4 pages, 2011.

[22] J. Barrett and J. A. Formaggio, "Resolving the reactor neutrino anomaly with the KATRIN neutrino experiment," 2011, http://arxiv.org/abs/1105.1326.

[23] A. Bungau, A. Adelmann, and J. R. Alonso, "Proposal for an electron antineutrino disappearance search using high-rate ${ }^{8} \mathrm{Li}$ production and decay," Physical Review Letters, vol. 109, no. 14, Article ID 141802, 5 pages, 2012.

[24] D. V. Forero and M. M. Guzzo, "Constraining nonstandard neutrino interactions with electrons," Physical Review D, vol. 84, no. 1, Article ID 013002, 2011.

[25] J. Appel, M. Bass, M. Bishai et al., "Physics Working group report to the LBNE Reconfiguration Steering Committee," 2012.

[26] K. Abe, T. Abe, H. Aihara et al., "Letter of intent: the HyperKamiokande experiment -detector design and physics potential," 2011, http://arxiv.org/abs/1109.3262.

[27] M. Gonzalez-Garcia, M. Maltoni, J. Salvado, and T. Schwetz, "Global fit to three neutrino mixing: critical look at present precision," Journal of High Energy Physics, vol. 2012, p. 123, 2012.

[28] Y. Abe, C. Aberle, J. dos Anjos et al., "Reactor $\bar{v}_{e}$ disappearance in the Double Chooz experiment," Physical Review D, vol. 86, no. 5, Article ID 052008, 21 pages, 2012.

[29] F. An, Q. An, J. Z. Bai et al., "Improved measurement of electron antineutrino disappearance at Daya Bay," Chinese Physics C, vol. 37, no. 1, Article ID 011001, 2013.
[30] J. Ahn, S. Chebotaryov, J. H. Choi et al., "Observation of reactor electron antineutrinos disappearance in the RENO experiment," Physical Review Letters, vol. 108, no. 19, Article ID 191802, 6 pages, 2012.

[31] K. Abe, N. Abgrall, Y. Ajima et al., "Indication of electron neutrino appearance from an accelerator-produced off-axis Muon neutrino beam," Physical Review Letters, vol. 107, no. 4, Article ID 041801, 8 pages, 2011.

[32] L. Montanet, K. Gieselmann, R. M. Barnett et al., "Review of particle properties," Physical Review D, vol. 50, no. 3, pp. 11731814, 1995.

[33] T. Bezerra, H. Furuta, F. Suekane, and T. Matsubara, "A global fit determination of effective $\Delta m_{31}^{2}$ from baseline dependence of reactor $\bar{v}_{e}$ disappearance," Physics Letters B, vol. 725, pp. 42715276, 2013.

[34] M. Gonzalez-Garcia, M. Maltoni, J. Salvado, and T. Schwetz, "Global fit to three neutrino mixing: critical look at present precision," Journal of High Energy Physics, vol. 2012, p. 123, 2012.

[35] J. Lesgourgues and S. Pastor, "Neutrino mass from cosmology," Advances in High Energy Physics, vol. 2012, Article ID 608515, 34 pages, 2012.

[36] G. Fogli, E. Lisi, A. Marrone, D. Montanino, A. Palazzo, and A. Rotunno, "Global analysis of neutrino masses, mixings, and phases: entering the era of leptonic CP violation searches," Physical Review D, vol. 86, no. 1, Article ID 013012, 10 pages, 2012.

[37] T. Ohlsson, "Status of non-standard neutrino interactions," Reports on Progress in Physics, vol. 76, no. 4, Article ID 044201, 2013.

[38] J. Conrad, C. Ignarra, G. Karagiorgi, M. Shaevitz, and J. Spitz, "Sterile neutrino fits to short-baseline neutrino oscillation measurements," Advances in High Energy Physics, vol. 2013, Article ID 163897, 26 pages, 2013.

[39] A. A. Aguilar-Arevalo, C. E. Anderson, A. O. Bazarko et al., "Neutrino flux prediction at MiniBooNE," Physical Review D, vol. 79, no. 7, Article ID 072002, 38 pages, 2009.

[40] K. Abe, N. Abgrall, H. Aihara et al., "T2K neutrino flux prediction," Physical Review D, vol. 87, no. 1, Article ID 012001, 34 pages, 2013.

[41] S. Kopp, "The NuMI neutrino beam at Fermilab," 2005, http://arxiv.org/abs/physics/0508001.

[42] G. Giacomelli, "The CNGS neutrino beam," Journal of Physics, vol. 116, no. 1, Article ID 012004, 2008.

[43] J. Formaggio and G. Zeller, "From eV to EeV: neutrino cross sections across energy scales," Reviews of Modern Physics, vol. 84, no. 3, pp. 1307-1341, 2012.

[44] M. Bishai, M. Diwan, S. Kettell et al., "Precision neutrino oscillation measurements using simultaneous high-power, lowenergy project-X beams," 2013, http://arxiv.org/abs/1307.0807.

[45] M. Gonzalez-Garcia, M. Maltoni, J. Salvado, and T. Schwetz, "Global fit to three neutrino mixing: critical look at present precision," Journal of High Energy Physics, vol. 2012, p. 123, 2012.

[46] C. Bemporad, G. Gratta, and P. Vogel, "Reactor-based neutrino oscillation experiments," Reviews of Modern Physics, vol. 74, no. 2, pp. 297-328, 2002.

[47] A. de Gouvea and J. Jenkins, "What can we learn from neutrino electron scattering?" Physical Review D, vol. 74, no. 3, Article ID 033004, 12 pages, 2006.

[48] R. L. Burman, M. E. Potter, and E. S. Smith, "Monte Carlo simulation of neutrino production by medium-energy protons in a beam stop," Nuclear Instruments and Methods in Physics A, vol. 291, no. 3, pp. 621-633, 1990. 
[49] J. M. Conrad and M. H. Shaevitz, "Multiple cyclotron method to search for CP violation in the neutrino sector," Physical Review Letters, vol. 104, no. 14, Article ID 141802, 2010.

[50] B. Pontecorvo, "Report P.D.-205 of the National Research Council of Canada Division of Atomic Energy (1946)," declassified and issued by the Atomic Energy Commission in 1949.

[51] B. Pontecorvo, Cambridge Monographs on Particle Physics, Nuclear Physics and Cosmology, Cambridge University Press, Cambridge, UK, 1991.

[52] C. L. Cowan Jr., F. Reines, F. B. Harrison, H. W. Kruse, and A. D. McGuire, "Detection of the free neutrino: a confirmation," Science, vol. 124, no. 3212, pp. 103-104, 1956.

[53] L. H. Thomas, "The paths of ions in the cyclotron I. Orbits in the magnetic field," Physical Review, vol. 54, no. 8, pp. 580-588, 1938.

[54] M. Seidel, Ch. Baumgarten, M. Bopp et al., "Towards the 2 MW Cyclotron and latest development at PSI," in Proceedings of the 19th International Conference on Cyclotrons and their Applications, 2010.

[55] M. Kase, E. Ikezawa, N. Fukunishi et al., "Present status of the riken ring cyclotron," in Proceedings of the 17th International Conference on Cyclotrons and their Applications, 2004.

[56] C. J. Oram, J. B. Warren, G. M. Marshall, and J. Doornbos, "Commissioning of a new low energy $\pi-\mu$ at triumf," Nuclear Instruments and Methods, vol. 179, no. 1, pp. 95-103, 1981.

[57] J. Blaser, H. Willax, and H. Gerber, "The sin ring cyclotron project status report," in Conference Proceedings C, pp. 556-565, 1969.

[58] R. L. Burman, R. L. Fulton, and M. Jakobson, "Design of the LAMPF Low-Energy Pion Channel," Nuclear Instruments and Methods, vol. 131, no. 1, pp. 29-38, 1975.

[59] V. Tishchenko, S. Battu, R. M. Carey et al., "Detailed report of the MuLan measurement of the positive Muon lifetime and determination of the Fermi constant," 2012, http://arxiv.org/abs/1211.0960.

[60] R. Allen, H. Chen, P. Doe et al., "Study of electron-neutrinoelectron elastic scattering at LAMPF," Physical Review D, vol. 47, no. 1, pp. 11-28, 1993.

[61] C. Athanassopoulos, L. B. Auerbach, R. L. Burman et al., "Evidence for $v_{\mu} \rightarrow v_{e}$ oscillations from the LSND experiment at the los alamos meson physics facility," Physical Review Letters, vol. 77, no. 15, pp. 3082-3085, 1996.

[62] B. Armbruster, I. M. Blair, B. A. Bodmann et al., "Upper limits for neutrino oscillations $\bar{v}_{\mu} \rightarrow \bar{v}_{e}$ from muon decay at rest," Physical Review D, vol. 65, no. 11, Article ID 112001, 16 pages, 2002.

[63] A. Bolozdynya, F. Cavanna, Y. Efremenko et al., "Opportunities for neutrino physics at the spallation neutron source: a white paper," 2012, http://arxiv.org/abs/1211.5199.

[64] L. Calabretta, "Utilization and reliability of high power proton accelerators," in Proceedings of the Nuclear Energy Agency (NEA Workshop), 1999.

[65] C. Rubbia, J. A. Rubio, S. Buono et al., "Conceptual design of a fast neutron operated high power energy amplifier," Tech. Rep. CERN/AT/95-44(ET), 1995.

[66] M. Reiser, Theory and Design of Charged Particle Beams, WileyVCH, New York, NY, USA, 2008.

[67] A. Adelmann, A. Gsell, C. Kraus et al., "The OPAL framework (object oriented parallel accelerator library) version 1.2.01," User's Reference Manual PSI-PR-08-02, Paul Scherrer Institut, Villigen, Switzerland, 2008-2013, http://amas.web.psi.ch/ docs/opal/opal_user_guide.pdf.
[68] J. J. Yang, A. Adelmann, M. Humbel, M. Seidel, and T. J. Zhang, "Beam dynamics in high intensity cyclotrons including neighboring bunch effects: model, implementation, and application," Physical Review Special Topics, vol. 13, no. 6, Article ID 064201, 2010.

[69] Y. J. Bi, A. Adelmann, R. Dölling et al., "Towards quantitative simulations of high power proton cyclotrons," Physical Review Special Topics, vol. 14, no. 5, Article ID 054402, 2011.

[70] F. Maimone, L. Celona, and F. Chines, "Status of the versatile ion source VIS," in Proceedings of the EPAC08-MOPC151 Conference, vol. C0806233, 2008.

[71] J. Alonso, "Relevance of IsoDAR and DAEdALUS to medical radioisotope production," 2012, http://arxiv.org/abs/arXiv: 1209.4925 .

[72] Cyclone-70, "Multiparticule high energy industrial cyclotron," Tech. Rep., 2009, http://www.iba-cyclotron-solutions .com/products-cyclo/cyclone-70.

[73] MYRRHA, http://www.euronuclear.org/e-news/e-news-21/myrrha.htm.

[74] S. K. Agarwalla and P. Huber, "Potential measurement of the weak mixing angle with neutrino-electron scattering at low energy," Journal of High Energy Physics, vol. 2011, p. 59, 2011.

[75] S. Agarwalla, J. Conrad, and M. Shaevitz, "Short-baseline neutrino oscillation waves in ultra-large liquid scintillator detectors ," Journal of High Energy Physics, vol. 2011, p. 85, 2011.

[76] D. Haxton and W. McCurdy, private email communication.

[77] A. Sen, "Production of Low Vibrational State Ions for Recombination Experiments," [Ph.D. thesis], University of Western Ontario, Ontario, Canada, 1985.

[78] A. A. Aguilar-Arevalo, B. C. Brown, L. Bugel et al., "Improved search for $\bar{v}_{\mu} \rightarrow \bar{v}_{e}$ oscillations in the MiniBooNE experiment," Physical Review Letters, vol. 110, no. 16, Article ID 161801, 6 pages, 2013.

[79] T. A. Mueller, D. Lhuillier, M. Fallot et al., "Improved predictions of reactor antineutrino spectra," Physical Review C, vol. 83, no. 5, Article ID 054615, 17 pages, 2011.

[80] P. Astier, D. Autiero, A. Baldisseri et al., "Search for $v_{\mu} \rightarrow v_{e}$ oscillations in the NOMAD experiment," Physics Letters $B$, vol. 570, no. 1-2, pp. 19-31, 2003.

[81] I. E. Stockdale, A. Bodek, F. Borcherding et al., "Search for muon neutrino and antineutrino oscillations in the mass range $15<\Delta \mathrm{m} 2<1,000 \mathrm{eV} 2 / \mathrm{c} 4$," Zeitschrift für Physik C Particles and Fields, vol. 27, no. 1, pp. 53-56, 1985.

[82] F. Dydak, G. J. Feldman, C. Guyot et al., "A search for $\nu \mu$ oscillations in the $\Delta \mathrm{m} 2$ range $0.3-90 \mathrm{eV} 2$, , Physics Letters $B$, vol. 134, no. 3-4, pp. 281-286, 1984.

[83] M. Sorel, J. M. Conrad, and M. H. Shaevitz, "Combined analysis of short-baseline neutrino experiments in the $(3+1)$ and $(3+2)$ sterile neutrino oscillation hypotheses," Physical Review D, vol. 70, no. 7, pp. 1-73004, 2004.

[84] A. Bungau, R. Barlow, M. Shaevitz, J. Conrad, and J. Spitz, "Target studies for the production of lithium8 for neutrino physics using a low energy cyclotron,” 2012, http://arxiv.org/ abs/1205.5790.

[85] P. Vogel and J. F. Beacom, "Angular distribution of neutron inverse beta decay, $\nu_{e}+p \rightarrow e^{+}+n$," Physical Review D, vol. 60, no. 5, Article ID 053003, 1999.

[86] A. Gando, Y. Gando, K. Ichimura et al., "Constraints on $\theta_{13}$ from a three-flavor oscillation analysis of reactor antineutrinos at KamLAND," Physical Review D, vol. 83, no. 5, Article ID 052002, 11 pages, 2011. 
[87] S. Abe, T. Ebihara, S. Enomoto et al., "Precision measurement of neutrino oscillation parameters with KamLAND," Physical Review Letters, vol. 100, no. 22, Article ID 221803, 2008.

[88] A. Gando, Y. Gando, H. Hanakago et al., "Reactor on-off antineutrino measurement with KamLAND," 2013, http://arxiv .org/abs/1303.4667.

[89] S. Abe, K. Furuno, A. Gando et al., "Measurement of the ${ }^{8}$ B solar neutrino flux with the KamLAND liquid scintillator detector," Physical Review C, vol. 84, no. 3, Article ID 035804, 2011.

[90] F. Reines, H. S. Gurr, and H. W. Sobel, "Detection of $\bar{\nu}_{e}$-e scattering," Physical Review Letters, vol. 37, no. 6, pp. 315-318, 1976.

[91] M. Deniz, S. Bilmis, and H. T. Wong, "Final results of $\bar{v}_{e} \rightarrow e$ scattering cross-section measurements and constraints on new physics," Journal of Physics, vol. 375, Article ID 042044, 2012.

[92] A. Gomonaǐ and I. Zapesochnyǐ, "Resonance excitation of bound and autoionization states of the ytterbium atom in the course of three-photon ionization," JETP Letters, vol. 57, no. 12, pp. 765-768, 1993.

[93] Z. Daraktchieva, C. Amsler, M. Avenier et al., "Final results on the neutrino magnetic moment from the MUNU experiment," Physics Letters B, vol. 615, no. 3-4, pp. 153-159, 2005.

[94] J. Conrad, M. Shaevitz, I. Shimizu, J. Spitz, M. Toups, and L. Winslow, "Precision $\bar{\nu}_{e}$-electron scattering measurements with IsoDAR to search for new physics".

[95] M. Baak, M. Goebel, A. Hoecker et al., "The electroweak fit of the standard model after the discovery of a new boson at the LHC," European Physical Journal C, vol. 72, p. 2205, 2012.

[96] G. P. Zeller, K. S. McFarland, T. Adams et al., "Precise determination of electroweak parameters in neutrino-nucleon scattering," Physical Review Letters, vol. 88, no. 9, Article ID 091802, 2002.

[97] J. Conrad, J. Link, and M. Shaevitz, "Precision measurement of $\sin ^{2} \theta_{W}$ at a reactor," Physical Review D, vol. 71, no. 7, Article ID 073013, 17 pages, 2005.

[98] S. Abe, K. Furuno, A. Gando et al., "Measurement of the ${ }^{8}$ B solar neutrino flux with the KamLAND liquid scintillator detector," Physical Review C, vol. 84, no. 3, Article ID 035804, 2011.

[99] H. Murayama and T. Yanagida, "Leptogenesis in supersymmetric standard model with right-handed neutrino," Physics Letters $B$, vol. 322, no. 4, pp. 349-354, 1994.

[100] E. Ma and U. Sarkar, "Neutrino masses and leptogenesis with heavy Higgs triplets," Physical Review Letters, vol. 80, no. 26, pp. 5716-5719, 1998.

[101] S. Davidson and A. Ibarra, "A lower bound on the right-handed neutrino mass from leptogenesis," Physics Letters B, vol. 535, no. 1-4, pp. 25-32, 2002.

[102] H. Nunokawa, S. Parke, and J. Valle, "CP violation and neutrino oscillations," Progress in Particle and Nuclear Physics, vol. 60, no. 2, pp. 338-402, 2008.

[103] R. Patterson, "The NOvA experiment: status and outlook," Nuclear Physics B, vol. 235-236, pp. 151-157, 2013.

[104] M. Aartsen, R. Abbasi, M. Ackermann et al., "PINGU sensitivity to the neutrino mass hierarchy," 2013, http://arxiv.org/abs/1306 .5846 .

[105] The DAEdALUS Collaboration, "A study of detector configurations for the DUSEL CP violation searches combining LBNE and DAEdALUS," 2010, http://arxiv.org/abs/1008.4967.

[106] M. Wurm, J. F. Beacom, L. B. Bezrukov et al., "The nextgeneration liquid-scintillator neutrino observatory LENA," Astroparticle Physics, vol. 35, no. 11, pp. 685-732, 2012.
[107] T. Akiri, D. Allspach, and M. Andrews, "The 2010 interim report of the long-baseline neutrino experiment collaboration physics working groups," 2011, http://arxiv.org/abs/1110.6249.

[108] A. J. Anderson, J. M. Conrad, E. Figueroa-Feliciano, K. Scholberg, and J. Spitz, "Coherent neutrino scattering in dark matter detectors," Physical Review D, vol. 84, no. 1, Article ID 013008, 2011. 

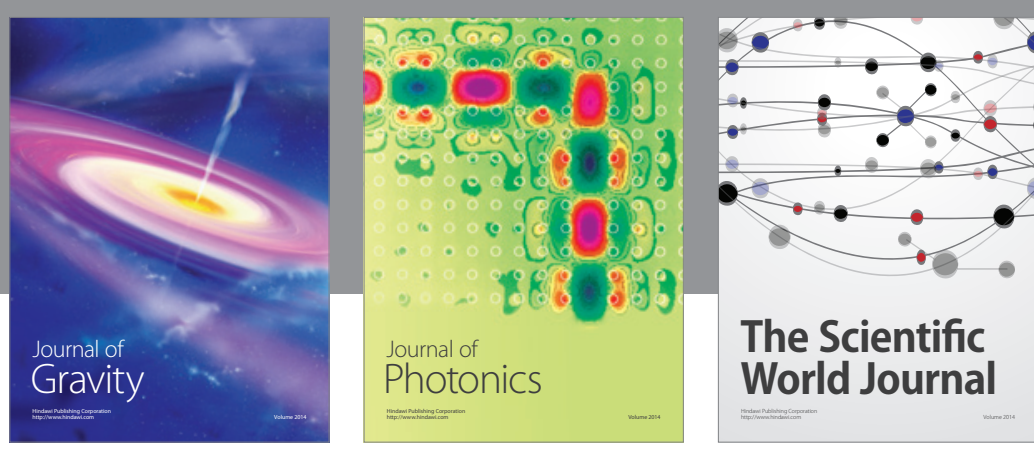

The Scientific World Journal
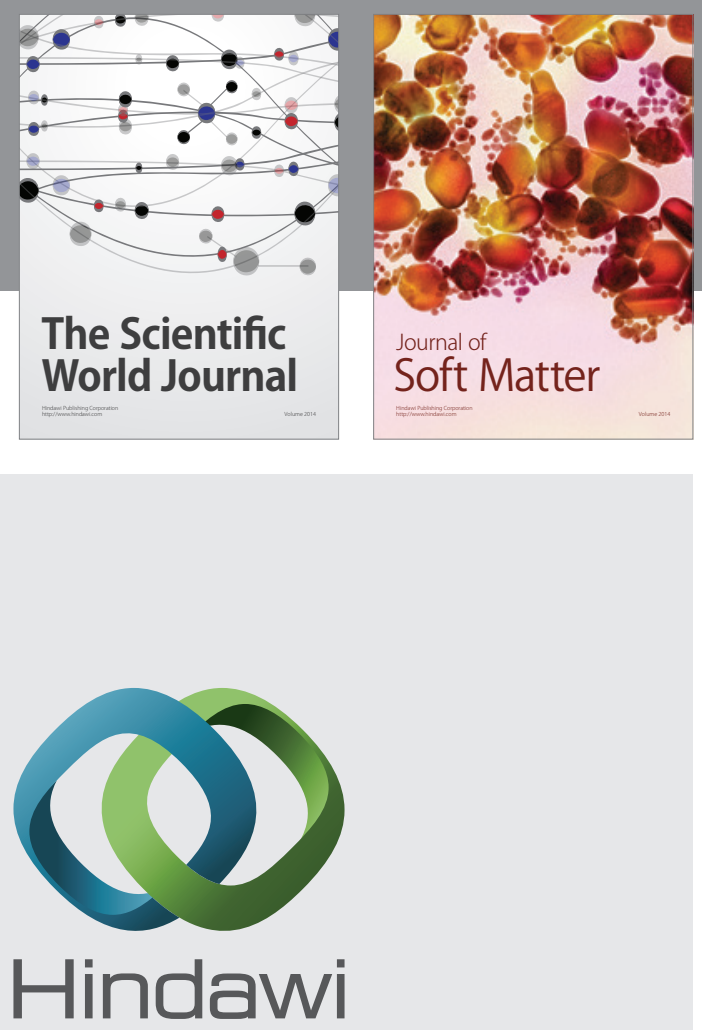

Submit your manuscripts at

http://www.hindawi.com

nternational Journal of

Statistical Mechanics
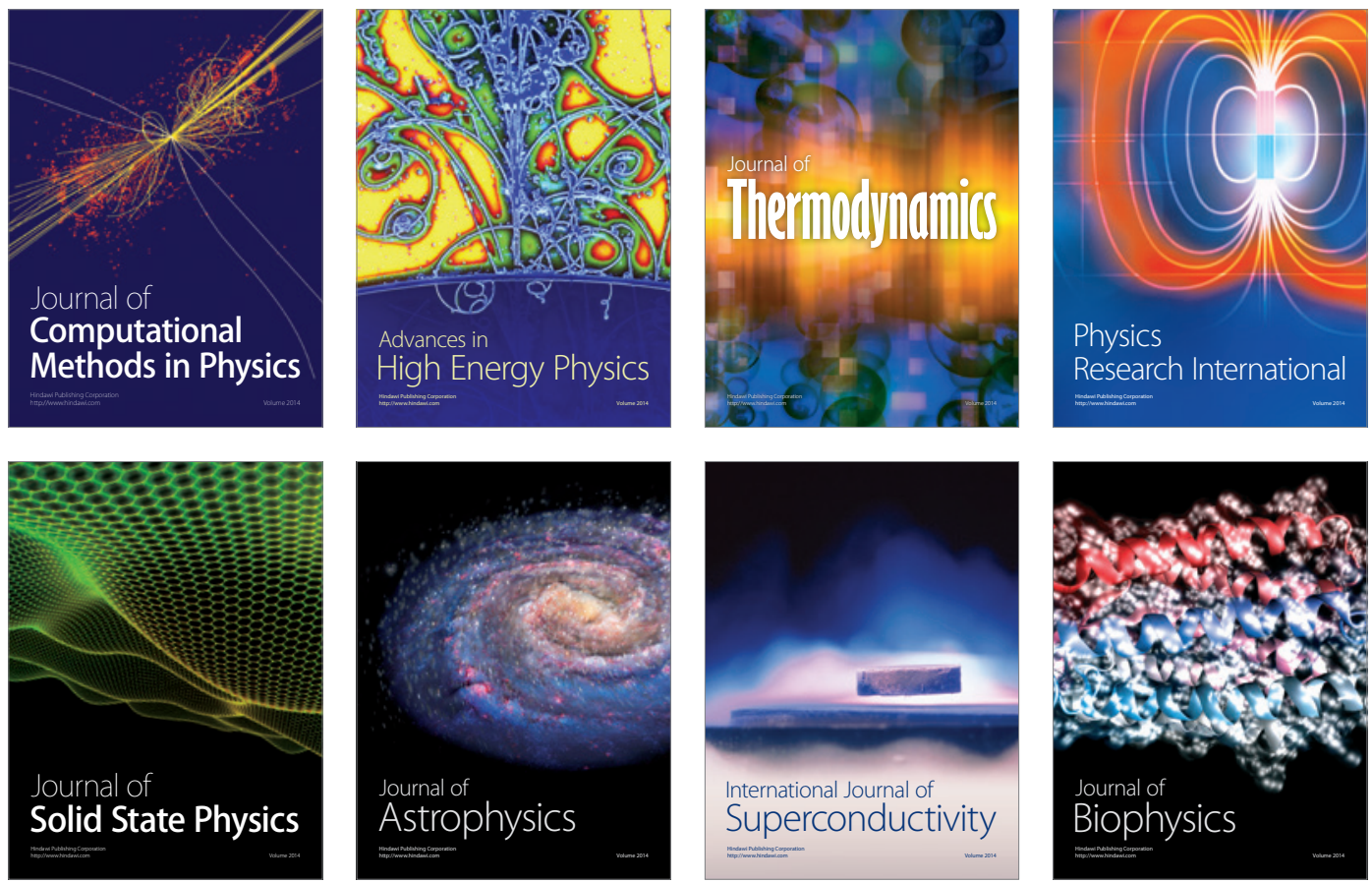
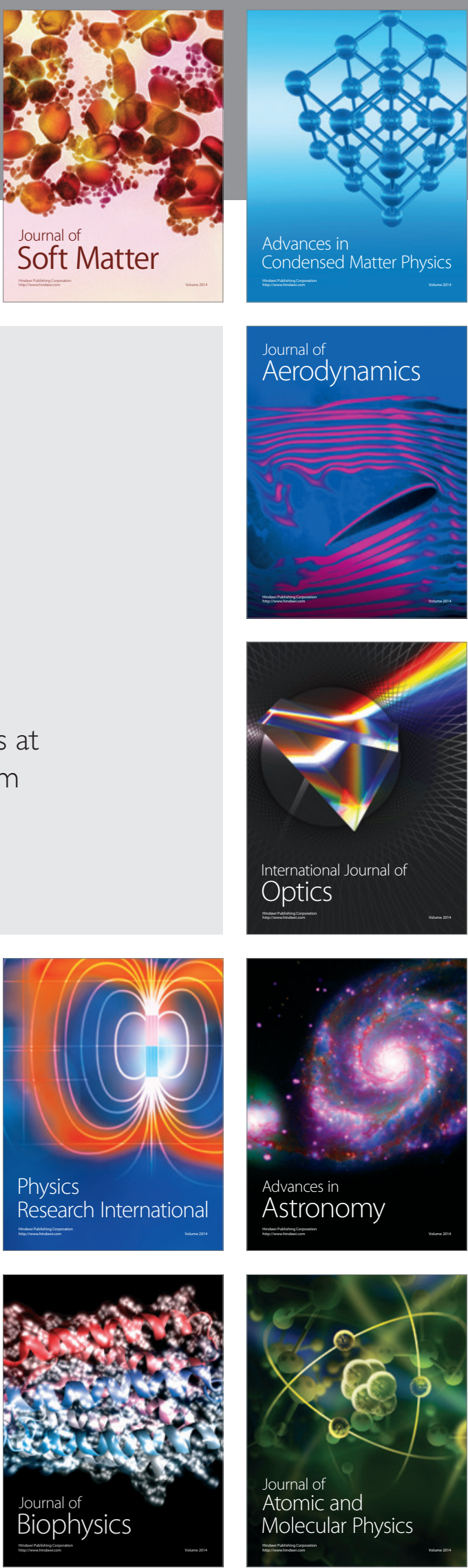